\title{
Local metric properties and regular stratifications of $p$-adic definable sets
}

\author{
Raf Cluckers, Georges Comte and François Loeser
}

\begin{abstract}
We study the geometry of germs of definable (semialgebraic or subanalytic) sets over a $p$-adic field from the metric, differential and measure geometric point of view. We prove that the local density of such sets at each of their points does exist. We then introduce the notion of distinguished tangent cone with respect to some open subgroup with finite index in the multiplicative group of our field and show, as it is the case in the real setting, that, up to some multiplicities, the local density may be computed on this distinguished tangent cone. We also prove that these distinguished tangent cones stabilize for small enough subgroups. We finally obtain the $p$-adic counterpart of the Cauchy-Crofton formula for the density. To prove these results we use the Lipschitz decomposition of definable $p$-adic sets of [5] and prove here the genericity of the regularity conditions for stratification such as $\left(w_{f}\right),(w),\left(a_{f}\right),(b)$ and $(a)$ conditions.
\end{abstract}

Mathematics Subject Classification (2010). Primary 03C10, 03C98, 12J10, 14B05, 32Sxx; Secondary 03C68, 11S80 14J17.

Keywords. $p$-adic definable sets, singularities, local density, Whitney stratification.

\section{Contents}

1 Preliminaries . . . . . . . . . . . . . . . 966

2 Local densities . . . . . . . . . . . . . . . . . . . . . . . . . . . . . . . . . . . . . . . . . . . 972

3 Tangent cones . . . . . . . . . . . . . . . . . . . . . 978

4 Existence of $\left(w_{f}\right)$-regular stratifications . . . . . . . . . . . . . . 984

5 Proof of Theorem 3.6.2 and the existence of distinguished tangent $\Lambda$-cones 990

6 A local Crofton formula . . . . . . . . . . . . . . . . . . 1001

References ...................... 1006

\section{Introduction}

Many fundamental results in motivic integration took their roots in the fertile soil provided by $p$-adic integration. The role played by analogy in the developments of motivic integration cannot be underestimated. The fundamental papers [19] and [18] took their source in their $p$-adic counterparts [13] and [16], respectively. The present 
paper is part of a larger project which is to develop a theory a local densities and tangent cones, and, more ambitiously, microlocal geometry, in a motivic framework. Since these objects were not considered yet over the $p$-adics, it was natural to start by figuring out the $p$-adic picture, which is of course already of interest for its own sake, in view of possible arithmetic applications. In fact, as the project advanced, we realized that the $p$-adic case already required some substantial issues to be settled while some unsuspected questions naturally arose. The present paper is devoted to the study of local metric properties of definable subsets of the $p$-adic affine space, with special stress on the local density of these subsets. It contains also results on tangent cones and existence of regular stratifications. We plan to consider the motivic analogues of the objects and results of this paper and of its companion [5] in subsequent work.

We shall start by recalling what is known in the real and complex context regarding the local density of (sub-)analytic sets. When $X_{a}$ is a germ at $a$ of a complex analytic subset $X$ of real dimension $d$ of the affine space $\mathbb{C}^{n}$, the local density $\Theta_{d}\left(X_{a}\right)$ of $X_{a}$, sometimes called in this setting the Lelong number of $X_{a}$, has been introduced by Lelong in [33] as the limit of volumes of the intersection of a representative $X$ of the germ with suitably renormalized balls around $a$, namely

$$
\Theta_{d}\left(X_{a}\right)=\lim _{r \rightarrow 0} \frac{\mu_{d}(X \cap B(a, r))}{\mu_{d}\left(B^{d}(0, r)\right)},
$$

where $B^{d}(0, r)$ is the real $d$-dimensional ball of centre 0 and radius $r>0$ and $\mu_{d}$ stands for $d$-dimensional volume. Lelong actually proved that the function

$$
r \mapsto \frac{\mu_{d}(X \cap B(a, r))}{r^{d}}
$$

decreases as $r$ goes to 0 , pointing out, long before this concept has been formalized, the tame behaviour of the local normalized volume of analytic sets. Two years after Lelong's pioneering paper, Thie proved in [41] that the local density of a complex analytic subset $X$ at a point $a$ is a positive integer by expressing it as a sum of local densities of the components, counted with multiplicities, of the tangent cone of $X$ at $a$. Finally, more than twenty years after Lelong's definition, Draper proved [20] that the local density is the algebraic multiplicity of the local ring of $X$ at $a$. The definition of local densities has been extended by Kurdyka and Raby to real subanalytic subsets of $\mathbb{R}^{n}$ in [32] (see also [31]). In fact, although the arguments in [32] and [31] which prove the existence of the density in the real subanalytic case were given before the notion of definable sets in o-minimal structures emerged, they apply to the real ominimal setting. A short proof of this existence result, again produced just before the concept of tame definable sets and involving the Cauchy-Crofton formula, may be found in the seminal paper [34] for semi-pfaffian sets. In [11] (Theorem 1.3), one can find a proof in the real o-minimal setting of the existence of the local density viewed 
as the higher term of a finite sequence of localized curvature invariants involving the Cauchy-Crofton formula and the theory of regular stratifications. Of course, in the real setting, the density is in general no longer a positive integer, but a non negative real number, and Kurdyka and Raby proved an appropriate extension of Thie's result by expressing again the local density in terms of the density of some components of the real tangent cone. The existence of the local density at each point of the closure of a subanalytic set is a manifestation of tameness of these sets near their singular points. Similarly, tameness in subanalytic geometry is also illustrated by the tame behavior of local density, viewed as a function of the base-point of the germ at which it is computed: it is actually proved in [12] that this function along a given global subanalytic set is a Log-analytic function, that is, a polynomial in subanalytic functions and their logarithms (see Siu's paper [40] for similar results in the complex case). Draper's result has been extended to the real setting by Comte in [10] in the following way. Recall that if $X$ is a complex analytic subset of the affine space of complex dimension $d$ at $x$, the algebraic multiplicity of the local ring of $X$ at $x$ is equal to the local degree of a generic linear projection $p: X \rightarrow \mathbb{C}^{d}$, that is, to the number of points near $x$ in a generic fiber of $p$. Over the reals, if $X$ is of local dimension $d$ at $x$, the number of points in fibers (near $x$ ) of a generic linear projection $p: X \rightarrow \mathbb{R}^{d}$ is not generically constant near $p(x)$ in general. The idea introduced in [10] to overcome this difficulty is to consider as a substitute for the local degree of $p$ the sum $d(p):=\sum_{i \in \mathbb{N}} i \cdot \theta_{i}$, where $\theta_{i}$ is the local density at $p(x)$ of the germ of the set of points in $\mathbb{R}^{d}$ over which the fiber of $p$ has exactly $i$ points near $x$. The so-called local Cauchy-Crofton formula proved in [10] states that the average along all linear projections $p$ of the degrees $d(p)$ is equal to the local density of $X$ at $x$ and can be considered as the real analogue of Draper's result. Finally, the complete multi-dimensional version of the local Cauchy-Crofton formula for real subanalytic sets is presented in [11] (Theorem 3.1), where the multi-dimensional substitute of the 0 -dimensional local degree $d(p)$ is obtained by considering the local Euler characteristic of generic multi-dimensional fibers, instead of the local number of points.

Now let $K$ be a finite extension of $\mathbb{Q}_{p}$ and $X$ be a definable subset (semi-algebraic or subanalytic) of $K^{n}$. Let $x$ be a point of $K^{n}$. When one tries to define the local density of $X$ at $x$ similarly to the archimedean case, one is faced with the problem, illustrated in 2.1, that the limit of local volumes in general no longer exists. It appears that the normalized volumes $v_{n}$ of $X$ in the balls $B(x, n):=\{w \in K \mid \operatorname{ord}(x-w) \geq$ $n\}$ has a periodic convergence, that is to say, there exists an integer $e>0$ such that for all $c=0, \ldots, e-1,\left(v_{c+m \cdot e}\right)_{m \in \mathbb{N}}$ has a limit $v_{c}$ in $\mathbb{Q}$ (see Proposition 2.2.3), with possibly $v_{c} \neq v_{c^{\prime}}$, for $c \neq c^{\prime}$. The reader having essentially in mind the real case is thus strongly encouraged to start reading this article by the example studied in 2.1 that emphasizes this phenomenon. We resolve that issue by using an appropriate renormalization device that leads us to express the mean value $\frac{1}{e} \cdot \sum_{c=0}^{e-1} v_{c}$ as the local density of $X$ at $x$. 
Another new issue occurring in the $p$-adic setting is the lack of a natural notion of a tangent cone. Unlike the real case where only the action of the multiplicative group $\mathbb{R}_{+}^{\times}$has to be considered, in the $p$-adic case, there seems to be no preferential subgroup of $K^{\times}$at hand. We remedy this by introducing, for each definable open subgroup of finite index $\Lambda$ in $K^{\times}$, a tangent cone $C_{x}^{\Lambda}$ at $x$ which is stable by homotheties in $\Lambda$, that is, which is a $\Lambda$-cone. One should note that such $\Lambda$-cones were already considered more than twenty years ago in the work of Heifetz on $p$-adic oscillatory integrals and wave front sets [25]. Nevertheless, we prove in Theorem 5.6.1 that, given a definable subset $X$, among these cones, some are distinguished as maximal for an inclusion property, and appear as the good tangent cones to be considered, in the sense that they capture the local geometry of our set. We are then able, by deformation to the tangent cone, to assign multiplicities to points in the tangent cone $C_{x}^{\Lambda}$.

Our main results regarding $p$-adic local densities are Theorem 3.6.2, which is a $p$-adic analogue of the result of Thie and Kurdyka-Raby, and Theorem 6.2.1, which is a $p$-adic analogue of Comte's local Cauchy-Crofton formula. An important technical tool in our proof of Theorem 3.6.2 is provided by Theorem 5.3.6 which allows us to decompose our definable set into Lipschitz graphs. Such a regular decomposition has been obtained in [5] and extends to the $p$-adic setting a real subanalytic result of [29]. In Section 4 we prove the existence of $\left(w_{f}\right)$-regular stratifications for definable $p$ adic functions, and consequently the existence of Thom's $\left(a_{f}\right)$-regular stratifications for definable $p$-adic functions, $(w)$-regular, or Verdier regular, stratifications, and Whitney's ( $b$ )-regular stratifications for $p$-adic definable sets (Theorem 4.2.5).

During the preparation of this paper, the authors have been partially supported by grant ANR-06-BLAN-0183. We also thank the Fields Institute of Toronto, where this paper was partly written, for bringing us exceptional working conditions.

\section{Preliminaries}

1.1. Definable sets over the $\boldsymbol{p}$-adics. Let $K$ be a finite field extension of $\mathbb{Q}_{p}$ with valuation ring $R$. We denote by ord the valuation and set $|x|:=q^{-\operatorname{ord}(x)}$ and $|0|=0$, with $q$ cardinality of the residue field of $K$. If $x=\left(x_{i}\right)$ is a point in $K^{m}$ and $n$ is an integer, we denote by $B(x, n)$ the ball in $K^{m}$ given by the conditions ord $\left(z_{i}-x_{i}\right) \geq n$, $1 \leq i \leq m$.

We recall the notion of (globally) subanalytic subsets of $K^{n}$ and of semi-algebraic subsets of $K^{n}$. Let $\mathscr{L}_{\text {Mac }}=\left\{0,1,+,-, \cdot,\left\{P_{n}\right\}_{n>0}\right\}$ be the language of Macintyre and $\mathscr{L}_{\text {an }}=\mathscr{L}_{\text {Mac }} \cup\left\{{ }^{-1}, \cup_{m>0} K\left\{x_{1}, \ldots, x_{m}\right\}\right\}$, where $P_{n}$ stands for the set of $n$th powers in $K^{\times}$, where ${ }^{-1}$ stands for the field inverse extended on 0 by $0^{-1}=0$, where $K\left\{x_{1}, \ldots, x_{m}\right\}$ is the ring of restricted power series over $K$ (that is, formal power series converging on $R^{m}$ ), and each element $f$ of $K\left\{x_{1}, \ldots, x_{m}\right\}$ is interpreted as 
the restricted analytic function $K^{m} \rightarrow K$ given by

$$
x \mapsto \begin{cases}f(x) & \text { if } x \in R^{m} \\ 0 & \text { else. }\end{cases}
$$

By subanalytic we mean $\mathscr{L}_{\text {an }}$-definable with coefficients from $K$ and by semi-algebraic we mean $\mathscr{L}_{\text {Mac }}$-definable with coefficients from $K$. Note that subanalytic, resp. semialgebraic, sets can be given by a quantifier free formula with coefficients from $K$ in the language $\mathscr{L}_{\text {Mac }}$, resp. $\mathscr{L}_{\text {an }}$.

In this section we let $\mathscr{L}$ be either the language $\mathscr{L}_{\mathrm{Mac}}$ or $\mathscr{L}_{\text {an }}$ and by $\mathscr{L}$-definable we will mean semi-algebraic, resp. subanalytic when $\mathscr{L}$ is $\mathscr{L}_{\text {Mac }}$, resp. $\mathscr{L}_{\text {an }}$. Everything in this paper will hold for both languages and we will give appropriate references for both languages when needed.

For each definable set $X \subset K^{n}$, let $\mathscr{C}(X)$ be the $\mathbb{Q}$-algebra of functions on $X$ generated by functions $|f|$ and ord $(f)$ for all definable functions $f: X \rightarrow K^{\times}$.

We refer to [22] and [17] for the definition of the dimension of $\mathscr{L}$-definable sets.

1.2. The $p$-adic measure. Suppose that $X \subset K^{n}$ is an $\mathscr{L}$-definable set of dimension $d \geq 0$. The set $X$ contains a definable nonempty open $K$-analytic submanifold $X^{\prime} \subset$ $K^{n}$ such that $X \backslash X^{\prime}$ has dimension $<d$, cf. [17]. There is a canonical $d$-dimensional measure $\mu_{d}$ on $X^{\prime}$ coming from the embedding in $K^{n}$, which is constructed as follows, cf. [39]. For each $d$-element subset $J$ of $\{1, \ldots, n\}$, with $j_{i}<j_{i+1}$, $j_{i}$ in $J$, let $d x_{J}$ be the $d$-form $d x_{j_{1}} \wedge \cdots \wedge d x_{j_{d}}$ on $K^{n}$, with $x=\left(x_{1}, \ldots, x_{n}\right)$ standard global coordinates on $K^{n}$. Let $x_{0}$ be a point on $X^{\prime}$ such that $x_{I}$ are local coordinates around $x_{0}$ for some $I \subset\{1, \ldots, n\}$. For each $d$-element subset $J$ of $\{1, \ldots, n\}$ let $g_{J}$ be the $\mathscr{L}$-definable function determined at a neighborhood of $x_{0}$ in $X^{\prime}$ by $g_{J} d x_{I}=d x_{J}$. There is a unique volume form $\left|\omega_{0}\right|_{X^{\prime}}$ on $X^{\prime}$ which is locally equal to $\left(\max _{J}\left|g_{J}\right|\right)\left|d x_{I}\right|$ around every point $x_{0}$ in $X^{\prime}$. Indeed, $\left|\omega_{0}\right|_{X^{\prime}}$ is equal to $\sup _{J}\left|d x_{J}\right|$. The canonical $d$-dimensional measure $\mu_{d}$ on $X^{\prime}$ (cf. [39], [37]), is the one induced by the volume form $\left|\omega_{0}\right|_{X^{\prime}}$. We extend this measure to $X$ by zero on $X \backslash X^{\prime}$ and still denote it by $\mu_{d}$.

1.3. Adding sorts. By analogy with the motivic framework, we now expand the language $\mathscr{L}$ to a three sorted language $\mathscr{L}^{\prime}$ having $\mathscr{L}$ as language for the valued field sort, the ring language $\mathbf{L}_{\text {Rings }}$ for the residue field, and the Presburger language $\mathbf{L}_{\mathrm{PR}}$ for the value group together with maps ord and $\overline{\mathrm{ac}}$ as in [8]. By taking the product of the measure $\mu_{m}$ with the counting measure on $k_{K}^{n} \times \mathbb{Z}^{r}$ one defines a measure still denoted by $\mu_{m}$ on $K^{m} \times k_{K}^{n} \times \mathbb{Z}^{r}$.

One defines the dimension of an $\mathscr{L}^{\prime}$-definable subset $X$ of $K^{m} \times k_{K}^{n} \times \mathbb{Z}^{r}$ as the dimension of its projection $p(X) \subset K^{m}$. If $X$ is of dimension $d$, one defines a 
measure $\mu_{d}$ on $X$ extending the previous construction on $X$ by setting

$$
\mu_{d}(W):=\int_{p(X)} p_{!}\left(\mathbf{1}_{W}\right) \mu_{d}
$$

with $p_{!}\left(\mathbf{1}_{W}\right)$ the function $y \mapsto \operatorname{card}\left(p^{-1}(y) \cap W\right)$.

For such an $X$, one defines $\mathscr{C}(X)$ as the $\mathbb{Q}$-algebra of functions on $X$ generated by functions $\alpha$ and $q^{-\alpha}$ with $\alpha: X \rightarrow \mathbb{Z}$ definable in $\mathscr{L}^{\prime}$. Note that this definition coincides with the previous one when $n=r=0$. Since $\mathscr{L}^{\prime}$ is interpretable in $\mathscr{L}$, the formalism developed in this section extends to $\mathscr{L}^{\prime}$-definable objects in a natural way.

1.4. $p$-adic cell decomposition. Cells are defined by induction on the number of variables

1.4.1 Definition. An $\mathscr{L}$-cell $A \subset K$ is a (nonempty) set of the form

$$
\left\{t \in K|| \alpha\left|\square_{1}\right| t-c\left|\square_{2}\right| \beta \mid, t-c \in \lambda P_{n}\right\},
$$

with constants $n>0, \lambda, c$ in $K, \alpha, \beta$ in $K^{\times}$, and $\square_{i}$ either $<$ or no condition. An $\mathscr{L}$-cell $A \subset K^{m+1}, m \geq 0$, is a set of the form

$$
\left\{(x, t) \in K^{m+1}|x \in D,| \alpha(x)\left|\square_{1}\right| t-c(x)\left|\square_{2}\right| \beta(x) \mid, t-c(x) \in \lambda P_{n}\right\},
$$

with $(x, t)=\left(x_{1}, \ldots, x_{m}, t\right), n>0, \lambda$ in $K, D=p_{m}(A)$ a cell where $p_{m}$ is the projection $K^{m+1} \rightarrow K^{m}, \mathscr{L}$-definable functions $\alpha, \beta: K^{m} \rightarrow K^{\times}$and $c: K^{m} \rightarrow K$, and $\square_{i}$ either $<$ or no condition, such that the functions $\alpha, \beta$, and $c$ are analytic on $D$. We call $c$ the center of the cell $A$ and $\lambda P_{n}$ the coset of $A$. In either case, if $\lambda=0$ we call $A$ a 0 -cell and if $\lambda \neq 0$ we call $A$ a 1 -cell.

In the $p$-adic semi-algebraic case, cell decomposition theorems are due to Cohen [9] and Denef [13], [15] and they were extended in [3] to the subanalytic setting where one can find the following version.

1.4.2 Theorem ( $p$-adic cell decomposition). Let $X \subset K^{m+1}$ and $f_{j}: X \rightarrow K$ be $\mathscr{L}$-definable for $j=1, \ldots, r$. Then there exists a finite partition of $X$ into $\mathscr{L}$-cells $A_{i}$ with center $c_{i}$ and coset $\lambda_{i} P_{n_{i}}$ such that

$$
\left|f_{j}(x, t)\right|=\left|h_{i j}(x)\right| \cdot\left|\left(t-c_{i}(x)\right)^{a_{i j}} \lambda_{i}^{-a_{i j}}\right|^{\frac{1}{n_{i}}}, \quad \text { for each }(x, t) \in A_{i},
$$

with $(x, t)=\left(x_{1}, \ldots, x_{m}, t\right)$, integers $a_{i j}$, and $h_{i j}: K^{m} \rightarrow K \mathscr{L}$-definable functions which are analytic on $p_{m}\left(A_{i}\right), j=1, \ldots, r$. If $\lambda_{i}=0$, we use the convention that $a_{i j}=0$.

Let us also recall the following lemma from [4]. 
1.4.3 Lemma. Let $X \subset K^{m+1}$ be $\mathscr{L}$-definable and let $G_{j}$ be functions in $\mathscr{C}(X)$ in the variables $\left(x_{1}, \ldots, x_{m}, t\right)$ for $j=1, \ldots, r$. Then there exists a finite partition of $X$ into $\mathscr{L}$-cells $A_{i}$ with center $c_{i}$ and coset $\lambda_{i} P_{n_{i}}$ such that each restriction $\left.G_{j}\right|_{A_{i}}$ is a finite sum of functions of the form

$$
\left|\left(t-c_{i}(x)\right)^{a} \lambda_{i}^{-a}\right|^{\frac{1}{n_{i}}} \operatorname{ord}\left(t-c_{i}(x)\right)^{s} h(x),
$$

where $h$ is in $\mathscr{C}\left(K^{m}\right)$, and $s \geq 0$ and $a$ are integers.

The following $p$-adic curve selection lemma is due to van den Dries and Scowcroft [22] in the semi-algebraic case and to Denef and van den Dries [17] in the subanalytic case. The statement is the $p$-adic counterpart of the semi-algebraic or subanalytic curve selection lemma over the reals.

1.4.4 Lemma (Curve selection). Let $A$ be a definable subset of $K^{n}$ and let $x$ be in $\bar{A}$. Then there exists a definable function $f=\left(f_{1}, \ldots, f_{n}\right): R \rightarrow K^{n}$ such that the $f_{i}$ are given by power series (over $K$ ) converging on $R$ such that $f(0)=x$, and such that $f(R \backslash\{0\}) \subset A$.

The following is a $p$-adic analogue of a classical lemma by Whitney (see [44]).

1.4.5 Lemma ( $p$-adic Whitney Lemma). Let $g: R \rightarrow K^{n}$ be a map given by $n$ analytic power series over $K$, converging on $R$, such that the map $g$ is nonconstant. Then, the limit $\ell \in \mathbf{P}^{n-1}(K)$ for $r \rightarrow 0$ of the lines $\ell_{r} \in \mathbf{P}^{n-1}(K)$ connecting $g(0)$ with $g(r)$ exists. Also the limit $\ell^{\prime}$ of the tangent lines $\ell_{r}^{\prime}:=\left\{g(r)+\lambda\left(\partial g_{1} / \partial r, \ldots, \partial g_{n} / \partial r\right)_{|r|}\right.$ $\lambda \in K\}$ for $r \rightarrow 0$ exists and $\ell^{\prime}=\ell$.

Proof. Since $g$ is nonconstant, for $r \neq 0$ close to 0 one has $g(r) \neq g(0)$ and $\left(\partial g_{1} / \partial r, \ldots, \partial g_{n} / \partial r\right)_{\mid r} \neq 0$, and hence, $\ell_{r}$ and $\ell_{r}^{\prime}$ are well defined for $r \neq 0$ close to 0 . We may suppose that $g(0)=0$ and that each of the $g_{i}$ is nonconstant. Write $g_{i}(r)=\sum_{j \geq 0} a_{i j} r^{j}$ with $a_{i j} \in K$ and for each $i$, let $k_{i}$ be the smallest index $j$ such that $a_{i j} \neq 0$. Then $k_{i}>0$ for each $i$ since $g(0)=0$. Let $k$ be the minimum of the $k_{i}$. Then clearly $\ell$ and $\ell^{\prime}$ are the same line $\ell$ connecting 0 and $\left(a_{1 k}, \ldots, a_{n k}\right) \neq 0$. Indeed, the line $\ell_{r}$ connects 0 and $g(r)$ which is equivalent to connecting 0 and $g(r) / r^{k}$; the point $g(r) / r^{k}$ converges to $\left(a_{1 k}, \ldots, a_{n k}\right)$ and thus $\ell_{r}$ converges to the line $\ell$. Likewise, the line $\ell_{r}^{\prime}$ connects 0 and $\left(\partial g_{1} / \partial r, \ldots, \partial g_{n} / \partial r\right)_{\mid r}$ which is equivalent to connecting 0 and

$$
\frac{1}{k r^{k}}\left(\partial g_{1} / \partial r, \ldots, \partial g_{n} / \partial r\right)_{\mid r}
$$

the point $\frac{1}{k r^{k}}\left(\partial g_{1} / \partial r, \ldots, \partial g_{n} / \partial r\right)_{\mid r}$ converges to $\left(a_{1 k}, \ldots, a_{n k}\right)$ and thus also $\ell_{r}^{\prime}$ converges to $\ell$ when $r \rightarrow 0$. 
1.5. Fix two integers $d \leq m$. Let $U$ be an open definable subset of $K^{d}$ and let $\varphi$ be a definable analytic mapping $U \rightarrow K^{m-d}$. We view the graph $\Gamma(\varphi)$ of $\varphi$ as a definable subset of $K^{m}$. Let $\varepsilon$ be a positive real number. We say that $\varphi$ is $\varepsilon$-analytic, if the norm $|D \varphi|=\max _{i, j}\left|\partial \varphi_{i} / \partial x_{j}\right|$ of the differential of $\varphi$ is less or equal than $\varepsilon$ at every point of $U$.

For $\varepsilon>0$, call a function $f: D \rightarrow K^{m}$ on a subset $D$ of $K^{n} \varepsilon$-Lipschitz when for all $x, y \in D$ one has

$$
|f(x)-f(y)| \leq \varepsilon|x-y| .
$$

The function $f$ is called locally $\varepsilon$-Lipschitz when for each $x \in D$ there exists an open subset $U$ of $K^{n}$ containing $x$ such that the restriction of $f$ to $U \cap D$ is $\varepsilon$-Lipschitz.

1.5.1 Lemma. Let $U$ be open in $K^{n}$ and $f: U \rightarrow K^{m}$ a function which is $\varepsilon$-analytic. Then $f$ is locally $\varepsilon$-Lipschitz.

Proof. Choose $u \in U$, and a basic neighborhood $U_{u}$ of $u$ in $U$ such that the component functions $f_{i}$ of $f$ are given by converging power series on $U_{u}$, where basic neighborhood means a ball of the form $c+\lambda R^{n}$ with $c \in K^{n}$ and $\lambda \in K^{\times}$. We may suppose that $U_{u}=R^{n}$, that $u=0$, and that $\varepsilon=1$. We may also assume that for each $i, j$, the partial derivative $\partial f_{j}(x) / \partial x_{i}$ is bounded in norm by 1 on $U_{u}$. Since $\left|\frac{\partial f_{j}(x)}{\partial x_{i}}(0)\right| \leq 1$, it follows that the linear term of $f_{j}$ in $x_{i}$ has a coefficient of norm $\leq 1$ for each $i, j$. By the convergence of the power series, the coefficients of the $f_{j}$ are bounded in norm, say by $N$, and we can put $U^{\prime}:=\left\{x \in R^{n}|| x \mid<1 / N\right\}$. Clearly $U^{\prime}$ contains $u=0$. By the non-archimedean property of the $p$-adic valuation, the restriction of $f$ to $U^{\prime}$ is 1-Lipschitz.

For more results related to Lipschitz continuity on the $p$-adics, see [5] or Theorem 5.3.6 below. The following lemma is a partial converse of Lemma 1.5.1, especially in view of the fact that any definable function is piecewise analytic.

1.5.2 Lemma. Let $U$ be a definable open in $K^{n}$ and let $f: D \rightarrow K^{m}$ be a definable analytic function which is locally $\varepsilon$-Lipschitz. Then $f$ is $\varepsilon$-analytic.

Proof. We proceed by contradiction. Suppose that $|D f|>\varepsilon$ at $u \in U$. Choose a basic neighborhood $U_{u}$ of $u$ in $U$ such that the component functions $f_{i}$ of $f$ are given by converging power series on $U_{u}$, (here again by basic neighborhood we mean a ball of the form $c+\lambda R^{n}$ with $c \in K^{n}$ and $\lambda \in K^{\times}$). We may suppose that $U_{u}=R^{n}$, that $u=0$, and that $\varepsilon=1$. By assumption, we have for some $i, j$ that $\left|\left(\partial f_{j}(x) / \partial x_{i}\right)(0)\right|>1$, hence, the linear term of $f_{j}$ in $x_{i}$ has a coefficient of norm strictly greater than 1 . By the convergence of the power series, the coefficients of the $f_{j}$ are bounded in norm, say by $N$, and thus for any $x$ in $\left\{x \in R^{n}|| x \mid<1 / N\right\}$ one has $|f(0)-f(x)|>|x|$ which contradicts the fact that $f$ is $\varepsilon$-Lipschitz with $\varepsilon=1$. 
The following is the $p$-adic analogue of Proposition 1.4 of [32].

1.5.3 Proposition. Let $X$ be a definable subset of dimension $d$ of $K^{m}$. For every $\varepsilon>0$, there exists a definable subset $Y$ of $X$ of dimension $<d, N(\varepsilon) \geq 0$, definable open subsets $U_{i}(\varepsilon)$ of $K^{d}$, for $1 \leq i \leq N(\varepsilon)$, definable, $\varepsilon$-analytic functions $\varphi_{i}(\varepsilon): U_{i}(\varepsilon) \rightarrow K^{m-d}$, with graphs $\Gamma_{i}(\varepsilon)$, and elements $\gamma_{1}, \ldots, \gamma_{N(\varepsilon)}$ in $\operatorname{GL}_{m}(R)$ such that the sets $\gamma_{i}\left(\Gamma_{i}(\varepsilon)\right)$ are all disjoint and contained in $X$, and

$$
X=\bigcup_{1 \geq i \geq N(\varepsilon)} \gamma_{i}\left(\Gamma_{i}(\varepsilon)\right) \cup Y .
$$

Proof. Up to taking a finite partition of $X$ into definable analytic manifolds and neglecting parts of dimension $<d$, we may suppose that $X$ is itself a definable analytic manifold of dimension $d$. A finite partition of the Grassmann manifold by sets of diameter less than $\varepsilon$ (take for instance the distance $\delta$ considered in Section 4.2) induces a partition of the tangent space of $X$ and induces in turn a partition of $X$. Let us consider one of these parts, say $X$ again. Fix $\gamma$ a linear transform in $\operatorname{GL}_{m}(R)$ such that for every $x \in X$, the distance from $\gamma\left(T_{x} X\right)$ to $K^{d} \times\{0\}^{m-d}$ is less than $\varepsilon$. Then by cell decomposition, one may assume that $\gamma(X)$ is a graph of an analytic definable map $f$ from an open set $U \subset K^{d}$ to $K^{m-d}$. Clearly this map is $\varepsilon$-analytic, and, since $\gamma$ is a linear isometry, $X$ is the graph of the $\varepsilon$-analytic map $\gamma^{-1} \circ f \circ \gamma$ from $\gamma^{-1}(U)$ to $\gamma^{-1}\left(\{0\}^{d} \times K^{m-d}\right)$.

1.5.4 Remark. Although in the statement of Proposition 1.5.3 the real $\varepsilon$ may be as small as we want, one can restrict to $\varepsilon=1$ to prove the main Theorems 3.6.2, 5.6.1 and 6.2.1.

The following lemma is classical, see, for example, [21] for the semi-algebraic case and, for example, [6] for the subanalytic case. Let $\mathscr{L}^{*}$ be the language $\mathscr{L}$ together with a function symbol for the field inverse on $K^{\times}$(extended by zero on zero), function symbols for each $n$ which stands for a (definable) $n$-th root picking function $\sqrt[n]{ }$ on the $n$-th powers (extended by zero outside the $n$-th powers), and for each degree $n$ a Henselian root picking function $h_{n}$ for polynomials of degree $n$ in the $n+1$ coefficients (extended by zero if the conditions of Hensel's Lemma are not fulfilled).

1.5.5 Lemma ([21], [6]). Let $f: D \subset K^{n} \rightarrow K^{m}$ be an LL-definable function. Then $D$ can be partitioned into finitely many definable pieces $D_{i}$ such that there are $\mathscr{L}^{*}$-terms $t_{i}$ with $f(x)=t_{i}(x)$ for each $i$ and each $x \in D_{i}$. 


\section{Local densities}

2.1. A false start. Let $X$ be a definable subset of $K^{m}$ of dimension $d$ and let $x$ be a point of $K^{m}$. Considering what is already known in the complex analytic and real o-minimal case, a natural way to define the local density of $X$ at $x=\left(x_{1}, \ldots, x_{m}\right)$ would be to consider the limit of $q^{n d} \mu_{d}(X \cap B(x, n))$, as $n \rightarrow \infty$. Unfortunately this naïve attempt fails as is shown by the following example that we present in detail in order to caution the reader not to rely too heavily on intuition coming from the real setting. Take $X$ the subset of points of even valuation in $K$ and $x=0$. Write $\pi_{K}$ for a uniformizer of $R$. The unit ball $B$ in $K$ being of measure 1, the ball $\pi_{K}^{\ell} \cdot B=B(0, \ell)$ of radius $q^{-\ell}$ has volume $q^{-\ell}$ and, by consequence, the sphere $\pi_{K}^{\ell} \cdot S=\pi_{K}^{\ell} \cdot B \backslash \pi_{K}^{\ell+1} \cdot B$ of radius $q^{-\ell}$ has volume $q^{-\ell}\left(1-q^{-1}\right)$. For $k \in \mathbb{N}$, let us first compute the volume of $X \cap B(0,2 k)$. The set $X \cap B(0,2 k)$ is the disjoint union of the spheres $\pi_{K}^{2 j} \cdot S$ for $j \geq k$ and thus has as volume

$$
\mu_{1}(X \cap B(0,2 k))=\left(1-q^{-1}\right)\left(q^{-2 k}+q^{-2 k-2}+\cdots\right)=\frac{q^{-2 k}}{1+q^{-1}} .
$$

On the other hand, the set $X \cap B(0,2 k-1)$ is also the disjoint union of the spheres $q^{2 j} \cdot S$ for $j \geq k$ and thus has as volume

$$
\mu_{1}(X \cap B(0,2 k-1))=\frac{q^{-2 k}}{1+q^{-1}} .
$$

We finally see that in this example the value of the $\operatorname{limit}_{\ell \rightarrow \infty} \frac{\mu_{1}(X \cap B(0, \ell))}{\mu_{1}(B(0, \ell))}$ depends on the parity of $\ell$, since

$$
\lim _{k \rightarrow \infty} q^{2 k} \mu_{1}(X \cap B(0,2 k))=\left(1+q^{-1}\right)^{-1}
$$

and

$$
\lim _{k \rightarrow \infty} q^{2 k-1} \mu_{1}(X \cap B(0,2 k-1))=(1+q)^{-1} .
$$

In our example one notices that the convergence of the ratio $\frac{\mu_{1}(X \cap B(0, \ell))}{\mu_{1}(B(0, \ell))}$ is 2-periodic and that one may recover the expected local density, which should be $\frac{1}{2}$, by taking the average of the two limits. To obviate the kind of difficulty presented by this example (the periodic convergence), we are led to introduce a regularization device that we shall explain now.

2.2. Mean value at infinity of bounded constructible functions. We will use the following elementary definition of the mean value at infinity of certain real valued functions on $\mathbb{N}$. 
2.2.1 Definition. Say that a function $h: \mathbb{N} \rightarrow \mathbb{R}$ has a mean value at infinity if there exists an integer $e>0$ such that

$$
\lim _{\substack{n \rightarrow \infty \\ n=c \bmod e}} h(n)
$$

exists in $\mathbb{R}$ for each $c=0, \ldots, e-1$ and in this case define the mean value at infinity of $h$ as the average

$$
\mathrm{MV}_{\infty}(h):=\frac{1}{e} \sum_{c=0}^{e-1} \lim _{\substack{n \rightarrow \infty \\ n=c \bmod e}} h(n) .
$$

Clearly the value $\mathrm{MV}_{\infty}(h)$ is independent of the choice of the modulus $e>0$.

Let $X$ be a definable subset of $K^{m}$, so that $X \times \mathbb{N}$ is a definable subset of $K^{m} \times \mathbb{Z}$. Say that a real valued function $g$ on $X \times \mathbb{N}$ is $X$-bounded if for every $x$ in $X$ the restriction of $g$ to $\{x\} \times \mathbb{N}$ is bounded (in the sense that $g(\{x\} \times \mathbb{N}$ ) is contained in a compact subset of $\mathbb{R})$. As has been indicated in the introduction and in the example of Section 2.1, for an $X$-bounded function $\varphi$ in $\mathscr{C}(X \times \mathbb{N})$ and $x \in X$, the function $\varphi_{x}: \mathbb{N} \rightarrow \mathbb{Q}, n \mapsto \varphi(x, n)$ may not have a unique limit for $n \rightarrow \infty$, but it may have a mean value at infinity $\operatorname{MV}_{\infty}\left(\varphi_{x}\right)$, as we will indeed show in Proposition 2.2.3. We will moreover show in Proposition 2.2.3 that $\operatorname{MV}_{\infty}\left(\varphi_{x}\right)$, considered as a function in $x \in X$, lies in $\mathscr{C}(X)$ and that $\operatorname{MV}_{\infty}\left(\varphi_{x}\right)$ can be calculated using a single integer $e$ as modulus when $x$ varies in $X$.

2.2.2 Lemma. Let $\varphi$ be in $\mathscr{C}(X \times \mathbb{N})$. Suppose that, for each $x \in X$, the function $\varphi_{x}: \mathbb{N} \rightarrow \mathbb{Q}, n \mapsto \varphi(x, n)$ has finite image. Then $\varphi_{x}$ has a mean value at infinity $\operatorname{MV}_{\infty}\left(\varphi_{x}\right)$ for each $x$. Moreover, there exist a definable function $b: X \rightarrow \mathbb{N}$ and an integer $e>0$ such that for all $c$ with $0 \leq c<e$ and all $x \in X$, the rational number $d_{c}(x):=\varphi(x, n)$ is independent of $n$ as long as $n \geq b(x)$ and $n \equiv c \bmod e$. Thus, for each $x \in X$, one has

$$
\operatorname{MV}_{\infty}\left(\varphi_{x}\right)=\frac{1}{e} \sum_{c=0}^{e-1} d_{c}(x)
$$

By consequence, the function $\mathrm{MV}_{\infty}\left(\varphi_{x}\right)$, considered as a function in $x \in X$, lies in $\mathscr{C}(X)$.

Proof. The lemma is a direct consequence of Lemma 1.4.3 and quantifier elimination in the three sorted language $\mathscr{L}^{\prime}$ of Section 1.3. Indeed, for $\varphi \in \mathscr{C}(X \times \mathbb{N})$ there exist, by Lemma 1.4 .3 and quantifier elimination in $\mathscr{L}^{\prime}$, a definable function $b: X \rightarrow \mathbb{N}$ and an integer $e>0$ such that for all $c$ with $0 \leq c<e$ one has

$$
\varphi(x, n)=\sum_{i=1}^{k} n^{\ell_{i}} q^{a_{i} n} h_{i c}(x)
$$


for all $x \in X$ and all $n$ with $n \geq b(x)$ and $n \equiv c \bmod e$, and where the $h_{i c}$ are in $\mathscr{C}(X)$ and $a_{i}$ in $\mathbb{Z}$. Clearly, by regrouping, we may suppose that the pairs $\left(\ell_{i}, a_{i}\right)$ are mutually different. But then, since $\varphi_{x}$ has finite image for each $x \in X$, one must find

$$
\varphi(x, n)=h_{j c}(x)
$$

for all $x \in X$ and all $n$ with $n \geq b(x)$ and $n \equiv c \bmod e$, where $j$ is such that $\left(\ell_{j}, a_{j}\right)=(0,0)$. Hence, one has $h_{j c}=d_{c}$ and we are done.

2.2.3 Proposition. Let $\varphi$ be in $\mathscr{C}(X \times \mathbb{N})$. Suppose that $\varphi$ is $X$-bounded. Then there exist $\varphi^{\prime}$ in $\mathscr{C}(X \times \mathbb{N})$ with $\lim _{n \rightarrow \infty} \varphi^{\prime}(x, n)=0$ for all $x \in X$ and such that the function

$$
g_{x}: \mathbb{N} \rightarrow \mathbb{Q}, \quad n \mapsto \varphi(x, n)-\varphi^{\prime}(x, n)
$$

has finite image. Clearly, the function $g: X \times \mathbb{N},(x, n) \mapsto g_{x}(n)$ lies in $\mathscr{C}(X \times \mathbb{N})$. Hence, $\mathrm{MV}_{\infty}\left(g_{x}\right)$ and $\mathrm{MV}_{\infty}\left(\varphi_{x}\right)$ exist and are equal and the function $\operatorname{MV}_{\infty}\left(\varphi_{x}\right)$, considered as a function in $x \in X$, lies in $\mathscr{C}(X)$. Also, if $\varphi \geq 0$ then $\operatorname{MV}_{\infty}\left(\varphi_{x}\right) \geq 0$ for all $x \in X$.

Proof. Write again $\varphi$ as in (2.2.1) for some integer $e$, where again the pairs $\left(\ell_{i}, a_{i}\right)$ are mutually different. Define $\varphi^{\prime}(x, n)$ as the partial sum

$$
\sum_{i \in I} n^{\ell_{i}} q^{a_{i} n} h_{i c}(x)
$$

for $x \in X$ and $n$ satisfying $n \geq b(x)$ and $n \equiv c \bmod e$, where $c=0, \ldots, e-1$, where $I$ consists of those $i$ with $a_{i}<0$. Extend $\varphi^{\prime}$ to the whole of $X \times \mathbb{N}$ by putting it equal to $\varphi$ for those $n$ with $n<b(x)$. Since $\varphi$ is $X$-bounded, one must have that $a_{i} \leq 0$ for all $i$, and, for those $i$ with $a_{i}=0$ one must have $\ell_{i}=0$. But then, we find

$$
g(x, n)=h_{j c}(x)
$$

for all $x \in X$ and all $n$ with $n \geq b(x)$ and $n \equiv c \bmod e$, where $j$ is such that $\left(\ell_{j}, a_{j}\right)=(0,0)$. For $n$ with $n<b(x)$ one clearly has $g(x, n)=0$. The conclusions now follow from Lemma 2.2.3.

2.3. Local densities. As already sketched in the introduction, we will define the local density of an $\mathscr{L}$-definable set $X \subset K^{m}$ at a point $x$ as the mean value at infinity of the renormalized measure of the intersection of $X$ with the sphere of radius $q^{-n}$ around $x$. At our disposal to show that this is well defined we have Proposition 2.2.3 and Lemma 2.3.1 below which guarantee the existence of the mean value at infinity. More generally, for a bounded function $\varphi$ in $\mathscr{C}(X)$, we extend $\varphi$ to $K^{m}$ by zero outside $X$ and we will define the density of $\varphi$ at any point $x \in K^{m}$ by a similar procedure, replacing the measure by an integral of $\varphi$ on a small sphere around $x$. 
Let $\varphi$ be a bounded function in $\mathscr{C}(X)$, meaning that the image of $\varphi$ is contained in a compact subset of $\mathbb{R}$. For $(x, n)$ in $K^{m} \times \mathbb{N}$ we set

$$
\gamma(\varphi)(x, n):=\int_{S(x, n) \cap X} \varphi(y) \mu_{d},
$$

where $S(x, n)$ is the sphere $\left\{y \in K^{m}|| x-y \mid=q^{-n}\right\}$ of radius $q^{-n}$ around $x$. Note that, by Lemma 2.3.3 below, one could as well work with balls around $x$ instead of spheres consequently in this section. By [14] for the semi-algebraic case and [3] for the subanalytic case, the function $\gamma(\varphi):(x, n) \mapsto \gamma(\varphi)(x, n)$ lies in $\mathscr{C}\left(K^{m} \times \mathbb{N}\right)$.

Suppose that $X$ is of dimension $d$. Then we renormalize $\gamma(\varphi)$ by dividing it by the volume of the $d$-dimensional sphere of corresponding radius and define the resulting function $\theta_{d}(\varphi)$ by

$$
\theta_{d}(\varphi)(x, n):=\frac{\gamma(\varphi)(x, n)}{\mu_{d}\left(S_{d}(n)\right)},
$$

where $S_{d}(n)$ is the $d$-dimensional sphere of radius $q^{-n}$, namely the set $\left\{w \in K^{d} \mid\right.$ $\left.|w|=q^{-n}\right\}$. Note that $S_{d}(n)$ has measure equal to $\left(1-q^{-d}\right) q^{-n d}$ and thus, $\theta_{d}(\varphi)$ lies in $\mathscr{C}\left(K^{m} \times \mathbb{N}\right)$.

The following lemma yields sufficient conditions for the mean value at infinity of $\theta_{d}(\varphi)$ to exist, in view of Proposition 2.2.3.

2.3.1 Lemma. Let $\varphi$ be a bounded function in $\mathscr{C}(X)$. Assume $X$ is of dimension $d$. Then the function $\theta_{d}(\varphi)$ lies in $\mathscr{C}\left(K^{m} \times \mathbb{N}\right)$ and is $K^{m}$-bounded.

Proof. That $\theta_{d}(\varphi)$ lies in $\mathscr{C}\left(K^{m} \times \mathbb{N}\right)$ is shown above, so we just have to show that $\theta_{d}(\varphi)$ is $K^{m}$-bounded. By the additivity of integrals and by cell decomposition, we may suppose that $X$ is a cell of dimension $d$. By changing the order of the coordinates if necessary and by Proposition 4.2.3, we may suppose that $X$ projects isometrically to the first $d$ coordinates of $K^{m}$. If now $M>0$ is such that $\varphi(y)$ lies in the real interval $[-M, M]$ for all $y \in X$, then it is clear by construction that $\theta_{d}(\varphi)(x)$ also lies in $[-M, M]$ for all $x \in K^{m}$.

It follows from Lemma 2.3.1 and Proposition 2.2.3 that if $\varphi$ is a bounded function in $\mathscr{C}(X)$ one can set

$$
\Theta_{d}(\varphi):=\operatorname{MV}_{\infty} \theta_{d}(\varphi),
$$

that is, for $x \in K^{m}, \Theta_{d}(\varphi)(x)$ is the mean value at infinity of the function $n \mapsto$ $\theta_{d}(\varphi)(x, n)$. By Proposition 2.2.3, the function $\Theta_{d}(\varphi)$ lies in $\mathscr{C}\left(K^{m}\right)$. For $x$ in $K^{m}$, we call $\Theta_{d}(\varphi)(x)$ the local density of $\varphi$ at $x$. More generally, if $\varphi$ is bounded on a neighborhood of some $x \in K^{m}$, then $\Theta_{d}(\varphi)(x)$ can be defined by first extending $\varphi$ by zero outside of this neighborhood and calculate its local density by the above definitions which is clearly independent of the choice of the neighborhood. One should also note that $\Theta_{d}(\varphi)(x)$ is zero when $x$ does not belong to the closure of $X$. 
2.3.2 Definition. Let $X$ be a definable subset of $K^{m}$ of dimension $d$ and let $x$ be a point in $K^{m}$. We call the rational number

$$
\Theta_{d}(X)(x):=\Theta_{d}\left(\mathbf{1}_{X}\right)(x)
$$

the local density of $X$ at $x$, where $\mathbf{1}_{X}$ is the characteristic function of $X$ which clearly lies in $\mathscr{C}\left(K^{m}\right)$.

Note that Definition 2.3.2 resembles the definition of the complex and real density as given in the introduction, where instead of the limit $\lim _{r \rightarrow 0}$ one takes $\mathrm{MV}_{\infty}$.

2.3.3 Lemma. Renormalizing with balls instead of with spheres yields the same local density functions $\Theta_{d}$. Precisely, for $\mathscr{L}$-definable $X$ of dimension $d$ and for $\varphi$ a bounded function in $\mathscr{C}(X)$ one has for $x \in K^{m}$

$$
\Theta_{d}(\varphi)=\operatorname{MV}_{\infty}\left(\theta_{d}^{\prime}(\varphi)\right)
$$

where

$$
\begin{gathered}
\theta_{d}^{\prime}(\varphi)(x, n):=\frac{\gamma^{\prime}(\varphi)(x, n)}{\mu_{d}\left(B_{d}(n)\right)}, \\
\gamma^{\prime}(\varphi)(x, n):=\int_{B(x, n) \cap X} \varphi(y) \mu_{d},
\end{gathered}
$$

and where $B_{d}(n)$ is the $d$-dimensional ball of radius $q^{-n}$, namely $\left\{w \in K^{d}|| w \mid \leq\right.$ $\left.q^{-n}\right\}$, and $B(x, n)$ is the ball $\left\{y \in K^{m}|| x-y \mid \leq q^{-n}\right\}$ around $x$ as defined in 1.1. In particular, $\theta_{d}^{\prime}(\varphi)$ lies in $\mathscr{C}\left(K^{m} \times \mathbb{N}\right)$ and is $K^{m}$-bounded and thus its $\mathrm{MV}_{\infty}$ is well defined.

Proof. That $\theta_{d}^{\prime}(\varphi)$ lies in $\mathscr{C}\left(K^{m} \times \mathbb{N}\right)$ and is $K^{m}$-bounded is proven as Lemma 2.3.1. We have to prove that $\Theta_{d}(\varphi)=\operatorname{MV}_{\infty}\left(\theta_{d}^{\prime}(\varphi)\right)$, that is, for $x \in K^{m}, \Theta_{d}(\varphi)(x)$ is the mean value at infinity of the function $n \mapsto \theta_{d}^{\prime}(\varphi)(x, n)$. It is clear that

$$
\gamma(\varphi)(x, n)=\gamma^{\prime}(\varphi)(x, n)-\gamma^{\prime}(\varphi)(x, n+1)
$$

and that

$$
\theta_{d}(\varphi)(x, n)=\frac{1}{\left(1-q^{-d}\right)}\left(\theta_{d}^{\prime}(\varphi)(x, n)-q^{-d} \theta_{d}^{\prime}(\varphi)(x, n+1)\right) .
$$

Now we are done by the following fact, which holds for any real constant $b \neq 1$. If a function $f: \mathbb{N} \rightarrow \mathbb{R}$ has a mean value at infinity, then so does $g: \mathbb{N} \rightarrow \mathbb{R}$, $n \mapsto \frac{1}{1-b}(f(n)-b f(n+1))$, and their mean values at infinity are equal. 
2.3.4 Example. Let us note that in the example of 2.1 of points of even valuation in $K$, one gets $\Theta_{1}(X)(0)=\frac{1}{2}$. More generally, if $\Lambda$ is a definable open subgroup of finite index $r$ in $K^{\times}$and $y$ is a point in $K^{\times}$, we have $\Theta_{1}(\Lambda y)(0)=\frac{1}{r}$. Indeed, it is easily checked that $\Theta_{1}(\Lambda y)(0)$ does not depend on $y$, hence if $y_{1}, \ldots, y_{r}$ is a set of representative of $K^{\times} / \Lambda$, we have $1=\Theta_{1}\left(\bigcup_{1 \leq i \leq r} \Lambda y_{i}\right)(0)=r \Theta_{1}(\Lambda)(0)$.

2.3.5 Proposition. Let $\varphi$ be a bounded function in $\mathscr{C}(X)$ and assume $X$ is of dimension $d$. Denote by $\tilde{\varphi}$ the extension of $\varphi$ by zero on $K^{m}$. Then the support of $\tilde{\varphi}-\Theta_{d}(\varphi)$ is contained in an $\mathscr{L}$-definable set of dimension $<d$.

Proof. Suppose $X \subset K^{m}$. Since for $x$ not in $\bar{X}$, for all sufficiently large $n$ one has that $\theta_{d}(\varphi)(x, n)=0$, the support of $\Theta_{d}(\varphi)$ is contained in the closure $\bar{X}$ of $X$ in $K^{n}$. After removing a subset of dimension $<d$ we may assume $X$ is a smooth subvariety and $\varphi$ is locally constant (for example after an iterated application of Lemma 1.4.3), in which case the result is clear.

2.4. For further use we shall give some basic properties of local densities.

2.4.1 Proposition. Let $X$ be definable subset of dimension $d$ of $K^{m}$. Then

$$
\Theta_{d}(X)(x)=\Theta_{d}(\bar{X})(x),
$$

where $\bar{X}$ denotes the closure of $X$.

Proof. Indeed, by additivity it is enough to prove that $\Theta_{d}(\bar{X} \backslash X)(x)=0$, which follows from the fact that $\Theta_{d}(Y)(x)=0$ when $Y$ is definable of dimension $<d$.

2.4.2 Proposition. Let $X$ be an $\mathscr{L}$-definable set of dimension $d$ and let $M>0$ be a constant. Consider a sequence offunctions $\varphi_{n}: X \rightarrow \mathbb{R}, n \in \mathbb{N}$ such that the function $(x, n) \mapsto \varphi_{n}(x)$ lies in $\mathscr{C}(X \times \mathbb{N})$ and such that $0 \leq \varphi_{n} \leq \varphi_{n+1} \leq \cdots \leq M$ for all $n$. Then the function $\varphi$ defined as $\sup \varphi_{n}$ lies in $\mathscr{C}(X)$ and is bounded. Moreover,

$$
\Theta_{d}(\varphi)(x)=\lim _{n} \Theta_{d}\left(\varphi_{n}\right)(x)
$$

and

$$
0 \leq \Theta_{d}\left(\varphi_{n}\right)(x) \leq \Theta_{d}\left(\varphi_{n+1}\right)(x)
$$

for each $n$ and $x$.

Proof. Clearly the function $\varphi$ is bounded and lies in $\mathscr{C}(X)$ by Proposition 2.2.3. Note that

$$
\gamma(\varphi)(x, m)=\lim _{n} \gamma\left(\varphi_{n}\right)(x, m)
$$


for each $m$ and $x$ by the Monotone Convergence Theorem. Hence, by the definition of $\theta_{d}$, also

$$
\theta_{d}(\varphi)(x, m)=\lim _{n} \theta_{d}\left(\varphi_{n}\right)(x, m)
$$

for each $m$ and $x$. Clearly

$$
0 \leq \gamma\left(\varphi_{n}\right) \leq \gamma\left(\varphi_{n+1}\right) \text { and } 0 \leq \theta_{d}\left(\varphi_{n}\right) \leq \theta_{d}\left(\varphi_{n+1}\right)
$$

for all $n$, on the whole of $X$, and hence

$$
0 \leq \Theta_{d}\left(\varphi_{n}\right)(x) \leq \Theta_{d}\left(\varphi_{n+1}\right)(x),
$$

by the definition of $\Theta_{d}$. Now the equality $\Theta_{d}(\varphi)(x)=\lim _{n} \Theta_{d}\left(\varphi_{n}\right)(x)$ follows from (2.4.1), (2.4.2), and the definitions of $\mathrm{MV}_{\infty}$ and $\Theta_{d}$, by changing the order of limits over $n$ and over $m$.

\section{Tangent cones}

3.1. Cones. We shall consider the set $\mathscr{D}$ of open finite index subgroups of $K^{\times}$(note that a finite index subgroup of $K^{\times}$is automatically open). We order $\mathscr{D}$ by inclusion. Note that for each $n>0$, the group $P_{n}$ of the $n$th powers in $K^{\times}$lies in $\mathscr{D}$, and any $\Lambda$ in $\mathscr{D}$ equals, as a set, a finite disjoint union of cosets of some $P_{n}$, see Lemma 3.1.1, and is thus $\mathscr{L}$-definable. We shall say a certain property (P) holds for $\Lambda$ small enough, if there exists $\Lambda_{0}$ in $\mathscr{D}$ such that $(\mathrm{P})$ holds for every $\Lambda \in \mathscr{D}$ contained in $\Lambda_{0}$.

Let $\Lambda$ be a subgroup of $K^{\times}$in $\mathscr{D}$. It acts naturally on $K^{n}$ by multiplicative translation $\lambda \cdot z:=\lambda z$, that is, by scalar multiplication on the vector space $K^{n}$. By a $\Lambda$-cone in $K^{n}$ we mean a subset $C$ of $K^{n}$ which is stable under the $\Lambda$-action, that is, $\Lambda \cdot C \subset C$ (note that this implies that $\Lambda \cdot C=C$ ). More generally, if $x \in K^{n}$, by a $\Lambda$-cone with origin $x$ we mean a subset $C$ of $K^{n}$ such that $C-x$ is stable under the $\Lambda$-action, where $C-x=\left\{t \in K^{n} \mid t+x \in C\right\}$. By a local $\Lambda$-cone with origin $x$, we mean a set of the form $C \cap B(x, n)$, with $C$ a $\Lambda$-cone with origin $x$ and $n$ in $\mathbb{N}$.

In Lemma 3.1.1 we describe all possible $\Lambda$-cones which are subsets of $K$, which turns out to be very similar to the real situation. In Section 3.2 we will show that definable sets in dimension 1 locally look like local $\Lambda$-cones (Lemma 3.2.1), and similarly in families of definable subsets of $K$ (Corollary 3.2.2). From 3.3 on we will define and study tangent cones and related objects, and formulate one of our main results on the relation between local densities of definable sets and of their tangent cones, viewed with multiplicities (the $p$-adic analogue of Thie's result), see Theorem 3.6.2.

3.1.1 Lemma. Let $C \subset K$ be a set. Then $C$ is a $\Lambda$-cone for some $\Lambda$ in $\mathscr{D}$ if and only if it is either the empty set or it is a finite disjoint union of sets of the form $\lambda P_{n}$ with $n>0$ and $\lambda \in K$. Hence, any cone $C \subset K$ is a definable set. 
Proof. Clearly the empty set is a $\Lambda$-cone for all $\Lambda$ and $\lambda \cdot P_{n}$ is a $\Lambda$-cone for $\Lambda \subset P_{n}$, and similarly for their finite unions. Now let $C$ be a nonempty $\Lambda$-cone for some $\Lambda$ in $D$. Either $C=\{0\}$ and we are done, or, up to replacing $C$ by $t C$ for some nonzero $t \in K$, we may suppose that $1 \in C$. But then $\Lambda \subset C$ and $C \backslash \Lambda$ is still a $\Lambda$-cone. Since the index of $\Lambda$ in $K^{\times}$is finite, it follows by a finite process that $C$ consists of a finite union of sets of the form $\mu \Lambda$ with $\mu \in K$. It remains to prove that $\Lambda$ itself is a finite disjoint union of sets of the form $\lambda P_{n}$, for some $n \in \mathbb{N}$ and some $\lambda \in K^{\times}$. Since $\Lambda$ is open and it must contain an open neighborhood $U=1+\mathcal{M}_{K}^{\ell}$ of 1 for some $\ell>0$ and with $\mathcal{M}_{K}$ the maximal ideal of $R$. Let $\pi_{K}$ be a uniformizer of $R$. Since $\Lambda$ has finite index in $K^{\times}$, there exists $n_{1}>0$ such that $\pi_{K}^{n_{1}}$ lies in $\Lambda$. Now let $n$ be a big enough multiple of $n_{1}$ such that $t^{n} \in U$ for all $t \in R^{\times}$. Then clearly $P_{n} \subset \Lambda$ and we are done since $P_{n}$ has finite index in $K^{\times}$and hence also in $\Lambda$.

3.2. Local conic structure of definable sets. Let $X$ be a definable subset of $K^{n}$ and let $x$ be a point in $K^{n}$. We denote by $\pi_{x}: K^{n} \backslash\{x\} \rightarrow \mathbf{P}^{n-1}(K)$ the function which to a point $z \neq x$ assigns the line containing $x$ and $z$. That is, for $x=0$, $\pi_{0}: K^{n} \backslash\{0\} \rightarrow \mathbf{P}^{n-1}(K)$ is the natural projection, and, for nonzero $x$, the map $\pi_{x}$ is the composition of $\pi_{0}$ with the translation $K^{n} \backslash\{x\} \rightarrow K^{n} \backslash\{0\}, y \mapsto y-x$. Furthermore we denote by

$$
\pi_{x}^{X}: X \backslash\{x\} \rightarrow \pi_{x}(X \backslash\{x\})
$$

the restriction of $\pi_{x}$ to $X \backslash\{x\}$.

3.2.1 Lemma. Let $Y$ be a definable subset of $K$. Then there exist $\Lambda$ in $\mathscr{D}$ and a definable function $\gamma: K \rightarrow \mathbb{N}$ such that $Y \cap B(y, \gamma(y))$ is a local $\Lambda$-cone with origin $y$, for all $y \in K$. If one writes $Y$ as a finite disjoint union of cells $Y_{i}$ with cosets $\lambda_{i} P_{n_{i}}$, then one can take $\Lambda=P_{N}$ with $N=\operatorname{lcm}\left(n_{i}\right)_{i}$.

Proof. The definability of $\gamma$ is not an issue by the definability of the conditions of being a local $\Lambda$-cone with origin $y$ and so on. By definition, a finite union of local $\Lambda$ cones is again a local $\Lambda$-cone. Hence, up to a finite partition using cell decomposition, we may suppose that $Y$ is a cell. Thus, $Y$ is of the form

$$
Y=\left\{t \in K|| \alpha\left|\square_{1}\right| t-c\left|\square_{2}\right| \beta \mid, t-c \in \lambda P_{n}\right\},
$$

for some constants $n>0, \lambda, c$ in $K, \alpha, \beta$ in $K^{\times}$, and $\square_{i}$ either < or no condition. Up to a transformation $t \mapsto t-c$ we may suppose that $c=0$. We may exclude the trivial case that $Y$ is a singleton, that is, we may suppose that $\lambda \neq 0$. Then $Y$ is open, and moreover, $Y$ is closed if and only if $\square_{2}$ is $<$. In the case that $\square_{2}$ is no condition, then the closure of $Y$ equals $Y \cup\{0\}$. Take $y \in K$. If $y$ lies outside the closure of $Y$, then $Y \cap B(y, n)$ is empty for sufficiently large $n$, and the empty set is a $\Lambda$-cone for any $\Lambda$ in $\mathscr{D}$. Also, if $y$ lies in the interior of $Y$, then $Y \cap B(y, n)$ is a ball around $y$ 
for sufficiently large $n$, and hence it is a local $\Lambda$-cone with origin $y$, for any $\Lambda$ in $\mathcal{D}$. Finally, if $y=0$ and $y$ lies in the closure of $Y$, then $Y \cap B(y, n)=\lambda P_{n} \cap B(y, n)$ for sufficiently large $n$, which is clearly a local $\Lambda$-cone with origin $y$ for any $\Lambda$ contained in $P_{n}$.

The following two corollaries of Lemma 3.2.1 are immediate.

3.2.2 Corollary. Let $Y$ be a definable subset of $K^{m+1}$. For each $x \in K^{m}$ write $Y_{x}$ for $\{t \in K \mid(x, t) \in Y\}$. Then there exist $\Lambda$ in $\mathcal{D}$ and a definable function $\gamma: K^{m+1} \rightarrow \mathbb{N}$ such that $Y_{x} \cap B(t, \gamma(x, t))$ is a local $\Lambda$-cone with origin $t$, for all $(x, t) \in K^{m+1}$. If one writes $Y$ as a finite disjoint union of cells $Y_{i}$ with cosets $\lambda_{i} P_{n_{i}}$, then one can take $\Lambda=P_{N}$ with $N=\operatorname{lcm}\left(n_{i}\right)_{i}$.

We will most often use the following variant of Corollary 3.2.2, which can be proved by working on affine charts.

3.2.3 Corollary. Let $X$ be a definable subset of $K^{n}$ and let $x$ be a point in $K^{n}$. Then there exist a definable function $\alpha_{x}: \mathbf{P}^{n-1}(K) \rightarrow \mathbb{N}$, that is, $\alpha_{x}$ is definable on each affine chart of $\mathbf{P}^{n-1}(K)$, and a group $\Lambda$ in $\mathcal{D}$ such that

$$
\left(\pi_{x}^{X}\right)^{-1}(\ell) \cap B\left(x, \alpha_{x}(\ell)\right)
$$

is a local $\Lambda$-cone with origin $x$ for every $\ell$ in $\mathbf{P}^{n-1}(K)$. Moreover, $\Lambda$ can be taken independently of $x$, and one can ensure that $(x, \ell) \mapsto \alpha_{x}(\ell)$ is a definable function from $K^{n} \times \mathbf{P}^{n-1}(K)$ to $\mathbb{N}$.

We shall call a subgroup $\Lambda$ in $\mathscr{D}$ satisfying the first condition in Corollary 3.2.3 adapted to $(X, x)$, and if moreover $\Lambda$ is adapted to $(X, x)$ for all $x \in K^{n}$, then we call $\Lambda$ adapted to $X$.

3.3. Tangent cones. Now, if $X$ is a definable subset of $K^{n}, x$ a point of $K^{n}$, and $\Lambda$ in $\mathscr{D}$, we define the tangent $\Lambda$-cone to $X$ at $x$ as

$$
\begin{aligned}
C_{x}^{\Lambda}(X):=\left\{u \in K^{n} \mid\right. & \text { for all } i>0 \text { there exist } z \in X, \lambda \in \Lambda \text { such } \\
& \text { that } \operatorname{ord}(z-x)>i \text { and } \operatorname{ord}(\lambda(z-x)-u)>i\} .
\end{aligned}
$$

By construction $C_{x}^{\Lambda}(X)$ is a closed, definable, $\Lambda$-cone, and, for any $n \in \mathbb{N}, C_{x}^{\Lambda}(X)=$ $C_{x}^{\Lambda}(X \cap B(x, n))$. Furthermore, for definable $X, Y \subset K^{n}$ and for $\Lambda^{\prime} \subset \Lambda$ in $\mathcal{D}$, one has

$$
\begin{aligned}
C_{x}^{\Lambda}(X \cup Y) & =C_{x}^{\Lambda}(X) \cup C_{x}^{\Lambda}(Y), \\
C_{x}^{\Lambda}(\bar{X}) & =C_{x}^{\Lambda}(X), \\
C_{x}^{\Lambda^{\prime}}(X) & \subset C_{x}^{\Lambda}(X) .
\end{aligned}
$$


Although the previous inclusion might be strict, $\operatorname{dim}\left(C_{x}^{\Lambda}(X)\right)$ does not depend on $\Lambda \in \mathscr{D}$ by Lemma 3.5.1. We comment some more on the previous inclusion in the following remarks.

3.3.1 Remark. Let $X$ be a local $\Lambda$-cone with origin $x$ in $K^{n}$. Thus, there exist $n$ in $\mathbb{N}$ and $C$ a $\Lambda$-cone with origin $x$ such that $X=C \cap B(x, n)$. In this case for any $\Lambda^{\prime} \subset \Lambda \in \mathcal{D}$, one has

$$
C_{x}^{\Lambda^{\prime}}(X)=C_{x}^{\Lambda}(X)(=C, \text { when } \mathrm{C} \text { is closed }) .
$$

Indeed, since $X=C \cap B(0, n)$, we have $C_{x}^{\Lambda^{\prime}}(X)=C_{x}^{\Lambda^{\prime}}(C)$. But we also have $C_{x}^{\Lambda^{\prime}}(C)=C_{x}^{\Lambda}(C)(=C$, when $C$ is closed $)$.

We indicate why $C_{x}^{\Lambda}(C) \subset C_{x}^{\Lambda^{\prime}}(C)$. Assuming $x=0$ for simplicity, let $u \in$ $C_{0}^{\Lambda}(C)$ and $i \in \mathbb{N}, z \in C, \lambda \in \Lambda$, with $\operatorname{ord}(z)>i$ and $\operatorname{ord}(\lambda z-u)>i$. We have $z \in C$ and thus $\lambda z \in C$. Now let $\lambda^{\prime} \in \Lambda^{\prime}$ small enough to ensure that $\operatorname{ord}\left(\lambda^{\prime} \lambda z\right)>i$. Then denoting $z^{\prime}=\lambda^{\prime} \lambda z$, one has $z^{\prime} \in C$. From $\operatorname{ord}\left(z^{\prime}\right)>i$ and ord $\left(\left(1 / \lambda^{\prime}\right) z^{\prime}-u\right)>i$, we see that $u \in C_{0}^{\Lambda^{\prime}}(C)$.

Finally we indicate why $C_{x}^{\Lambda}(C)=C$, when $C$ is closed. As $C$ is stable by the $\Lambda$-action, the inclusion $C \subset C_{x}^{\Lambda}(C)$ is obvious. On the other hand, assuming again $x=0$, if $u \in C_{x}^{\Lambda}(C)$, for all $i \in \mathbb{N}$, there exist $z \in C$ and $\lambda \in \Lambda$ such that $\operatorname{ord}(z)>i$ and $\operatorname{ord}(\lambda z-u)>i$. We can then construct a sequence of points $\lambda z \in C$ with limit $u$, this shows that $u \in C$, since $C$ is closed.

3.3.2 Remark. When $X$ is a definable subset of $K$ and $x$ a point of $K$, by Lemma 3.2.1, $X$ is a local $\Lambda$-cone at $x$ with origin $x$, for some $\Lambda \in \mathcal{D}$. By the above remark, for every $\Lambda^{\prime} \subset \Lambda$, and still in the one-dimensional case that $X \subset K$, one has $C_{x}^{\Lambda^{\prime}}(X)=C_{x}^{\Lambda}(X)$.

We cannot expect in general that for $X$ a definable subset of $K^{n}, n>1, X$ is a local $\Lambda$-cone for some $\Lambda \in \mathscr{D}$, but one may at least ask, as it is the case for $n=1$, whether the stability property "there exists $\Lambda \in \mathscr{D}$ such that for any $\Lambda^{\prime} \in \mathscr{D}, \Lambda^{\prime} \subset \Lambda$, one has $C_{x}^{\Lambda^{\prime}}(X)=C_{x}^{\Lambda}(X)$ " still holds for $n>1$. The answer to that question is yes, as we shall show in Theorem 5.6.1.

3.4. More on $\varepsilon$-analytic functions. The following is the $p$-adic analogue of Proposition 1.7 of [32].

3.4.1 Proposition. Let $f: U \rightarrow K^{n-d}$ be a definable $\varepsilon$-analytic function on a nonempty open subset $U$ of $K^{d}, 0 \leq d \leq n$. Let $\Gamma$ be the graph of $f$ and let $z$ be in $\bar{\Gamma}$. Then, for any group $\Lambda$ in $\mathscr{D}$

$$
C_{z}^{\Lambda}(\Gamma) \subset\left\{(x, y) \in K^{d} \times K^{n-d}|| y|\leq \varepsilon| x \mid\right\} .
$$

Proof. We may suppose that $z=0$. Choose $\Lambda$ in $\mathcal{D}$. Since

$$
C_{0}^{\Lambda}(\Gamma) \subset C_{0}^{K^{\times}}(\Gamma),
$$


by definition of $C_{0}^{\Lambda}(\cdot)$, we may assume that $\Lambda=K^{\times}$. We may also suppose that $\varepsilon=1$, after rescaling. Suppose by contradiction that there is $\left(x_{0}, y_{0}\right)$ in $C_{0}^{K^{\times}}(\Gamma)$ with $\left|y_{0}\right|>\left|x_{0}\right|+\delta$ for some $\delta>0$. Let $\Gamma^{\prime}$ be the intersection of $\Gamma$ with the open subset $\left\{(x, y) \in K^{d+(n-d)}|| y|>| x \mid+\delta\right\}$. By our assumption on $\left(x_{0}, y_{0}\right)$ and by the definition of $C_{0}^{K^{\times}}(\Gamma)$, the set $\Gamma^{\prime}$ is nonempty and 0 lies in $\bar{\Gamma}^{\prime} \backslash \Gamma^{\prime}$. Apply the Curve Selection Lemma 1.4.4 to the set $\Gamma^{\prime}$ and the point 0 . This way we find power series $g_{i}$ over $K$ in one variable for $i=1, \ldots, n$, converging on $R$, such that $g(0)=0$ and $g(R \backslash\{0\}) \subset \Gamma^{\prime} \backslash\{0\}$. But this is in contradiction with Lemma 1.4.5. Indeed, the tangent line $\ell_{r}^{\prime}$ at $r \neq 0$ is of the form $g(r)+K \cdot t_{r}$ with some $t_{r} \in K^{n}$ satisfying $\left|y\left(t_{r}\right)\right| \leq\left|x\left(t_{r}\right)\right|$ by $\varepsilon$-analyticity of $f$ and the chain rule for differentiation, where $x\left(t_{r}\right)=\left(t_{r 1}, \ldots, t_{r d}\right)$ and $y\left(t_{r}\right)=\left(t_{r d+1}, \ldots, t_{r n}\right)$. Hence, the limit $\ell_{0}^{\prime}$ of the $\ell_{r}^{\prime}$ for $0 \neq r \rightarrow 0$ is of the same form $g(0)+K \cdot t_{0}$ for some $t_{0} \in K^{n}$ with $\left|y\left(t_{0}\right)\right| \leq\left|x\left(t_{0}\right)\right|$. On the other hand, the line $\ell_{r}$ for $r \neq 0$ connecting $g(0)$ with $g(r)$ is of the form $g(r)+K \cdot u_{r}$ with some $u_{r} \in K^{n}$ satisfying $\left|y\left(u_{r}\right)\right|>\left|x\left(u_{r}\right)\right|+\delta$. Hence, the limit line $\ell_{0}$ of the $\ell_{r}$ for $r \rightarrow 0$ has the same description, which contradicts Lemma 1.4.5 and the description of $\ell_{0}^{\prime}$.

3.4.2 Corollary. With the data and the notation of Proposition 3.4.1, let $x$ be in $\bar{U}$. Then there are only finitely many points in $\bar{\Gamma}$ which project to $x$ under the coordinate projection $K^{d} \times K^{n-d} \rightarrow K^{d}$.

Proof. Suppose by contradiction that there are infinitely many such points. Then the dimension of $\bar{\Gamma} \cap\left(\{x\} \times K^{n-d}\right)$ is $>0$. Thus, there exists $z \in \bar{\Gamma}$ such that $C_{z}^{\Lambda}(\bar{\Gamma} \cap$ $\left.\left(\{x\} \times K^{n-d}\right)\right)$ is of dimension $>0$, which is in contradiction to Proposition 3.4.1.

3.5. Deformation to the tangent cone. Let $X$ be a definable subset of $K^{n}$ and let $x$ be a point of $K^{n}$. Fix a subgroup $\Lambda$ in $\mathscr{D}$. We consider the definable set $\mathscr{D}(X, x, \Lambda)$ in $K^{n} \times \Lambda$ defined as

$$
\mathcal{D}(X, x, \Lambda):=\{(z, \lambda) \mid x+\lambda z \in X\}
$$

and its closure

$$
\overline{\mathcal{D}(X, x, \Lambda)}
$$

in $K^{n} \times K$. In $\overline{\mathcal{D}(X, x, \Lambda)}$ one finds back the cone $C_{x}^{\Lambda}(X)$. Indeed, one has $\overline{\mathcal{D}(X, x, \Lambda)} \cap\left(K^{n} \times\{0\}\right)=C_{x}^{\Lambda}(X) \times\{0\}$, which we identify with $C_{x}^{\Lambda}(X)$.

3.5.1 Lemma. If $X$ is of dimension $d$, then $\mathcal{D}(X, x, \Lambda)$ is of dimension $d+1$ and $C_{x}^{\Lambda}(X)$ is of dimension $\leq d$. Moreover, $\operatorname{dim}\left(C_{x}^{\Lambda}(X)\right)$ does not depend on the choice of $\Lambda \in \mathcal{D}$. 
Proof. We may suppose that $X$ is nonempty. Consider the projection

$$
p: \mathscr{D}(X, x, \Lambda) \longrightarrow X, \quad(z, \lambda) \longmapsto x+\lambda z .
$$

Since $p$ is surjective and has fibers of dimension 1 , we get that $\mathscr{D}(X, x, \Lambda)$ is of dimension $d+1$. The cone $C_{x}^{\Lambda}(X)$ is contained in $\{0\} \cup(\overline{\mathscr{D}(X, x, \Lambda)} \backslash \mathscr{D}(X, x, \Lambda))$. Hence, $C_{x}^{\Lambda}(X)$ is of dimension $\leq d$. The last statement follows from

$$
C_{x}^{\Lambda}(X)=\bigcup_{i} \mu_{i} C_{x}^{\Lambda^{\prime}}(X),
$$

whenever $\Lambda^{\prime} \subset \Lambda$ is in $\mathcal{D}$ and when one writes $\Lambda$ as a finite union of cosets $\bigcup_{i} \mu_{i} \Lambda^{\prime}$ of $\Lambda^{\prime}$ in $\Lambda$.

3.6. Multiplicities on the tangent cones. Let $X$ be a definable subset of $K^{n}$ of dimension $d$, let $x$ be a point of $K^{n}$, and let $\Lambda$ be in $\mathcal{D}$. To each point $z$ on the cone $C_{x}^{\Lambda}(X)$ we will associate a rational number $S C_{x}^{\Lambda}(X)(z)$, called the multiplicity of $(X, x)$ at $z$ with respect to $\Lambda$.

Define the function

$$
S C_{x}^{\Lambda}(X): C_{x}^{\Lambda}(X) \rightarrow \mathbb{Q}
$$

as the function sending $z$ to

$$
\left[K^{\times}: \Lambda\right] \Theta_{d+1}\left(\mathbf{1}_{\mathfrak{D}(X, x, \Lambda)}\right)(z, 0)
$$

with $\left[K^{\times}: \Lambda\right]$ the index of $\Lambda$ in $K^{\times}$, and with $\mathbf{1}_{\mathscr{D}(X, x, \Lambda)}$ the characteristic function of $\mathscr{D}(X, x, \Lambda)$. The function $S C_{x}^{\Lambda}(X)$ is called the specialization of $X$ at $x$ with respect to $\Lambda$.

The following lemma gives an indication that $S C_{x}^{\Lambda}(X)$ captures much local information of $(X, x)$; this principle will find a strong and precise form in Theorem 3.6.2 below.

3.6.1 Lemma. The function $S C_{x}^{\Lambda}(X)$ lies in $\mathscr{C}\left(C_{x}^{\Lambda}(X)\right)$. Moreover

$$
\Theta_{d+1}\left(\mathbf{1}_{\mathscr{D}(X, x, \Lambda)}\right)(z, 0)=0
$$

for z outside $C_{x}^{\Lambda}(X)$.

Proof. The function $\mathbf{1}_{\mathscr{D}(X, x, \Lambda)}$ is in $\mathscr{C}\left(K^{n} \times K\right)$ since $\mathscr{D}(X, x, \Lambda)$ is a definable set, and thus, also $\Theta_{d+1}\left(\mathbf{1}_{\mathscr{D}(X, x, \Lambda)}\right)$ lies in $\mathscr{C}\left(K^{n} \times K\right)$. For definable sets $A \subset B$, the restriction of a function in $\mathscr{C}(B)$ to $A$ automatically lies in $\mathscr{C}(A)$, hence, $S C_{x}^{\Lambda}(X)$ lies in $\mathscr{C}\left(C_{x}^{\Lambda}(X)\right)$. The second statement follows from the fact that the support of $\Theta_{d+1}\left(\mathbf{1}_{\mathscr{D}(X, x, \Lambda)}\right)$ is contained in the closure of $\mathcal{D}(X, x, \Lambda)$, which is contained in $\left(K^{n} \times K^{\times}\right) \cup\left(C_{x}^{\Lambda}(X) \times\{0\}\right)$. 
More generally, if $\varphi$ is a function in $\mathscr{C}(X)$ which is bounded near $x$, we define the specialization $v_{x}^{\Lambda}(\varphi)$ of $\varphi$ at $x$ with respect to $\Lambda$ in the following way. First define a function $\psi$ on $K^{n} \times K$ by $\psi(z, \lambda):=\varphi(x+\lambda z)$ on $\mathscr{D}(X, x, \Lambda)$ and by zero elsewhere. Then one defines the function

$$
v_{x}^{\Lambda}(\varphi): C_{x}^{\Lambda}(X) \rightarrow \mathbb{Q}
$$

as the function sending $z$ to $\left[K^{\times}: \Lambda\right] \Theta_{d+1}(\psi)(z, 0)$. Note that, similarly as in Lemma 3.6.1, $v_{x}^{\Lambda}(\varphi)$ lies in $\mathscr{C}\left(C_{x}^{\Lambda}(X)\right)$ and that $\Theta_{d+1}(\psi)(z, 0)=0$ for $z$ outside $C_{x}^{\Lambda}(X)$. We recover $S C_{x}^{\Lambda}(X)$ since $v_{x}^{\Lambda}\left(\mathbf{1}_{X}\right)=S C_{x}^{\Lambda}(X)$.

The following result, which will be proved in Section 5, states that the local density can be computed on the tangent cone with multiplicities, for $\Lambda$ small enough.

3.6.2 Theorem. Let $X$ be a definable subset of $K^{n}$ and let $x$ be a point of $K^{n}$. For $\Lambda$ small enough

$$
\Theta_{d}(X)(x)=\Theta_{d}\left(S C_{x}^{\Lambda}(X)\right)(0) .
$$

More generally, let $\varphi$ be a function in $\mathscr{C}(X)$ which is bounded near $x$. For $\Lambda$ small enough

$$
\Theta_{d}(\varphi)(x)=\Theta_{d}\left(v_{x}^{\Lambda}(\varphi)\right)(0)
$$

\section{Existence of $\left(w_{f}\right)$-regular stratifications}

4.1. In his study of stability of the topological type of mappings, R. Thom introduced the regularity condition $\left(a_{f}\right)$ in [42], p. 274, as a relative version of condition $(a)$ of Whitney. The existence of $\left(a_{f}\right)$-regular stratifications was proved in the complex analytic case by H. Hironaka in [27] (Corollary 1, Section 5) using resolution of singularities, under the assumption "sans éclatement" which is always satisfied for functions. One can find proofs of the existence of $\left(a_{f}\right)$ stratifications in the real subanalytic case in [32], where the Puiseux Theorem with parameters of Pawłucki (see [38]) is used, and for o-minimal structures on the field of real numbers in [35].

The stronger condition $\left(w_{f}\right)$, the relative version of the so-called condition $(w)$ of Verdier (see [43]), was studied in the complex setting, for instance, in [26]. In the real subanalytic setting, it has been proved that $\left(w_{f}\right)$ stratifications exist by K. Bekka in [1], K. Kurdyka and A. Parusiński in [30] using Puiseux's Theorem with parameters, and finally by Ta Lê Loi in [36] for definable functions in some o-minimal structures over the real field (the o-minimal structure has to be polynomially bounded for the existence of $\left(w_{f}\right)$-regular stratifications, but need not to be so for the existence of $\left(a_{f}\right)$-regular stratifications). 
4.2. Let us now recall the definitions of $\left(w_{f}\right)$ and $\left(a_{f}\right)$-regular stratifications. Let $X$ be a definable subset of $K^{n}$, and let $\left(X^{j}\right)_{j \in\{1, \ldots, k\}}$ be a finite, definable and analytic stratification of $X$ satisfying the so-called frontier condition

$$
X^{i} \cap \overline{X^{j}} \neq \emptyset \Longrightarrow X^{i} \subset \overline{X^{j}},
$$

where definable and analytic means that the strata $X^{j}$ are definable, $K$-analytic manifolds. Let $S$ be a definable subset of $K$ and let $f: X \rightarrow S$ be a definable continuous mapping such that for any $j \in\{1, \ldots, k\}, f_{\mid X^{j}}$ is analytic and of constant rank (being 0 or 1). For $j \in\{1, \ldots, k\}$ and $x \in X^{j}$, let us denote by $T_{x} X_{f}^{j}$ the tangent space at $x$ of the fiber $f_{\mid X^{j}}^{-1}(f(x))$ of $f_{\mid X^{j}}$. Then one says that the pair of strata $\left(X^{i}, X^{j}\right)$ satisfies condition $\left(a_{f}\right)$ at a point $x_{0} \in X^{i} \subset \overline{X^{j}}$ if and only if for any sequence $\left(x_{r}\right)_{r \in \mathbb{N} \backslash\{0\}}$ of points of $X^{j}$ converging to $x_{0}$ and such that the sequence $\left(T_{x_{r}} X_{f}^{j}\right)_{r \in \mathbb{N} \backslash\{0\}}$ converges in the appropriate Grassmann manifold, one has

$$
\lim _{r \rightarrow \infty} \delta\left(T_{x_{0}} X_{f}^{i}, T_{x_{r}} X_{f}^{j}\right)=0,
$$

where $\delta(\cdot, \cdot)$ is a natural distance between linear subspaces of $K^{n}$ as defined below. Further, one says that the pair $\left(X^{i}, X^{j}\right)$ of strata satisfies condition $\left(w_{f}\right)$ at $x_{0}$ if and only if there exist a constant $C$ and a neighborhood $W_{x_{0}}$ of $x_{0}$ in $K^{n}$ such that for any $x \in \mathcal{W}_{x_{0}} \cap X^{i}$ and any $y \in \mathcal{W}_{x_{0}} \cap X^{j}$, one has

$$
\delta\left(T_{x} X_{f}^{i}, T_{y} X_{f}^{j}\right) \leq C \cdot|x-y| .
$$

In both definitions, $\delta\left(V, V^{\prime}\right)$ denotes the distance between two linear subspaces $V$ and $V^{\prime}$ of $K^{n}$ such that $\operatorname{dim}(V) \leq \operatorname{dim}\left(V^{\prime}\right)$, and is defined by

$$
\delta\left(V, V^{\prime}\right)=\sup _{v \in V,|v|=1}\left\{\inf _{v^{\prime} \in V^{\prime},\left|v^{\prime}\right|=1}\left|v-v^{\prime}\right|\right\}=\sup _{v \in V,|v|=1} \operatorname{dist}\left(v, S^{V^{\prime}}(0,1)\right),
$$

with $S^{V^{\prime}}(0,1)$ the unit sphere around 0 of $V^{\prime}$.

4.2.1 Remark. We have $\delta\left(V, V^{\prime}\right)=0$ if and only if $V \subset V^{\prime}$. Also, for any $V^{\prime \prime} \subset V^{\prime}$ such that $\operatorname{dim}(V) \leq \operatorname{dim}\left(V^{\prime \prime}\right), \delta\left(V, V^{\prime \prime}\right) \geq \delta\left(V, V^{\prime}\right)$.

One says that the stratification $\left(X^{j}\right)_{j \in\{1, \ldots, k\}}$ is $\left(a_{f}\right)$-regular, respectively $\left(w_{f}\right)$ regular if any pair $\left(X^{i}, X^{j}\right)$ of strata is $\left(a_{f}\right)$-regular, respectively $\left(w_{f}\right)$-regular at any point of $X^{i}$. And finally one says that the stratification $\left(X^{j}\right)_{j \in\{1, \ldots, k\}}$ is $(a)$-regular, respectively $(w)$-regular, if it is $\left(a_{f}\right)$-regular, respectively $\left(w_{f}\right)$-regular, for $S$ a point in $K$.

One starts the proof of the existence of $w_{f}$-regular stratifications with the key Lemma 4.2.4 (see [36], Lemma 1.8 for its real version). But before stating this 
lemma, let us introduce as in [5] (Definition 3.9) the notion of jacobian property for definable functions and recall from [5] that this property is in a sense a generic one (see Proposition 3.10 of [5] or Proposition 4.2.3 below). This will be used in the proof of Lemma 4.2.4.

4.2.2 Definition. Let $F: B \rightarrow B^{\prime}$ a definable function with $B, B^{\prime} \subset K$. We say that $F$ has the jacobian property if the following conditions hold all together:

(i) $F$ is a bijection and $B, B^{\prime}$ are balls,

(ii) $F$ is $C^{1}$ on $B$,

(iii) $\operatorname{ord}\left(\frac{\partial F}{\partial x}\right): B \rightarrow \mathbb{Z}$ is constant (and finite) on $B$,

(iv) for all $x, y \in B$ with $x \neq y$, one has

$$
\operatorname{ord}\left(\frac{\partial F}{\partial x}\right)+\operatorname{ord}(x-y)=\operatorname{ord}(F(x)-F(y)) .
$$

It is proved in a much more general setting in [7], Theorem 6.3.7, that the jacobian property is generic for definable mappings, which in our setting gives the following statement.

4.2.3 Proposition. Let $Y \subset K^{m}$ and $X \subset K \times Y$ be definable sets for some $m \in \mathbb{N}$. Let $M: X \rightarrow K$ be definable. Then there exists a finite partition of $X$ into definable subsets $X_{k}$ such that for each $y \in Y$, the restriction $M(\cdot, y): x_{1} \mapsto M\left(x_{1}, y\right)$ of $M$ to $\left\{x_{1} \in K \mid\left(x_{1}, y\right) \in X_{k}\right\}$ is either injective or constant.

Let us then assume, for simplicity, that on $X, M(\cdot, y)$ is injective. Then there exists a finite partition of $X$ into cells $A_{k}$ over $Y$ such that for each $y \in Y$ and each ball $B$ such that $B \times\{y\}$ is contained in $A_{k}$, there is a (unique) ball $B^{\prime}$ such that the map $M_{\mid B}: B \rightarrow B^{\prime}, x_{1} \mapsto M\left(x_{1}, y\right) \in B^{\prime}$ has the jacobian property.

Now we state and prove the key lemma used in the proof of Theorem 4.2.5.

4.2.4 Lemma. Let $M: \Omega \rightarrow K$ be a definable and differentiable function on an open definable subset $\Omega$ of $K^{m} \times K$ for some $m \geq 0$. Assume that $\bar{\Omega} \cap\left(K^{m} \times\{0\}\right)$ has a nonempty interior $U$ in $K^{m}$. Assume furthermore that $M$ is bounded on $\Omega$. Then there exist a nonempty open definable subset $V \subset U$ in $K^{m}$, an integer $\alpha>0$ and a constant $d \in K^{\times}$such that for all $x \in V$ and all $t$ with $\operatorname{ord} t>\alpha$ and $(x, t) \in \Omega$

$$
\left\|D_{x} M_{(x, t)}\right\| \leq|d| .
$$

In this lemma and later on, $D_{x} M_{(x, t)}$ means $\left(\partial M(x, t) / \partial x_{1}, \ldots, \partial M(x, t) / \partial x_{m}\right)$, and analogously, $D_{x_{1}} M_{(x, t)}$ means $\partial M(x, t) / \partial x_{1}$ and so on, and $\|\cdot\|$ denotes the maximum of the component norms.) 
Proof. Let us denote $(x, t)=\left(x_{1}, \ldots, x_{m}, t\right)=\left(x_{1}, y\right)$ the standard coordinates on $K^{m} \times K=K \times K^{m}$, where $y=\left(x_{2}, \ldots, x_{m}, t\right)$. (We will apply cell decomposition and related results sometimes with $x_{1}$ and sometimes with $t$ as special variable.) By the cell decomposition theorem (with special variable $t$ ) we can finitely partition $\Omega$ such that on each part $A$ such that $\bar{A}$ has nonzero intersection with $K^{m} \times\{0\}$ one has

$$
\left|D_{x_{i}} M_{(x, t)}\right|=\left\|D_{x} M_{(x, t)}\right\|=|c(x)| \cdot|\lambda t|^{a}
$$

for some $a \in \mathbb{Q}$, some $\lambda \in K^{\times}$, some $i \in\{1, \ldots, m\}$, and some definable function $c$. If all these exponents $a$ are nonnegative, then we are done since the $|c|$, as well as the boundary functions bounding $|t|$ from below in the cell descriptions are constant on small enough open subsets $V \subset U$. Let us assume that a particular $a$ is negative, say on a cell where $\left|D_{x_{1}} M_{(x, t)}\right|=\left\|D_{x} M_{(x, t)}\right\|$. By Proposition 4.2 .3 (with special variable $\left.x_{1}\right)$ applied to $M:\left(x_{1}, y\right) \mapsto M\left(x_{1}, y\right)$ there exists a finite number of cells $A_{k}$ partitioning $\Omega$ such that for each $y \in K^{m}$ and each ball $B$ with $B \times\{y\} \subset A_{k}$, $B \ni x_{1} \mapsto M\left(x_{1}, y\right)$ has the jacobian property. We necessarily have one of these cells $A_{k}$ such that $\overline{A_{k}} \cap\left(K^{m} \times\{0\}\right)$ has nonempty interior in $K^{m}$. We may assume by the cell decomposition theorem (with special variable $t$ ) that $A_{k}$ contains a subset $B_{1} \times B^{\prime} \times W$ with $B_{1}$ an open ball in the $x_{1}$ line, $B^{\prime}$ a Cartesian product of $m-1$ balls and $W$ an open definable subset of $K^{\times}$such that $0 \in \bar{W}$. Then, for any $y=\left(x_{2}, \ldots, x_{m}, t\right) \in B^{\prime} \times W$, by the jacobian property, the one-dimensional volume $\mu_{1}\left(M\left(B_{1} \times\{y\}\right)\right)$ equals $\mu_{1}\left(B_{1}\right) \cdot|c(x)| \cdot|t|^{a}$. Considering that $t$ can approach 0 while $|c(x)|$ stays constant and that $M$ is a bounded mapping, this is a contradiction.

We can finally prove our result concerning $\left(w_{f}\right)$-regular stratifications.

4.2.5 Theorem. Let $X$ be a definable subset of $K^{n}, S$ a definable subset of $K$ and $f: X \rightarrow S$ a definable continuous function. Then there exists a (finite) analytic definable stratification of $X$ which is $\left(w_{f}\right)$-regular. In particular definable subsets of $K^{n}$ also admit $\left(a_{f}\right),(w)$ and $(a)$-regular definable stratifications.

Proof. We proceed similarly as in [36]. Let $\left(X^{j}\right)_{j \in\{1, \ldots, k\}}$ be an analytic and definable stratification of $X$ such that the $f_{\mid X^{j}}$ are analytic and such that the rank of $f_{\mid X^{j}}$ is constant for all $j \in\{1, \ldots, k\}$. The set $w_{f}\left(X^{i}, X^{j}\right)$ of points $x \in X^{i}$ at which the pair $\left(X^{i}, X^{j}\right)$ is $\left(w_{f}\right)$-regular being a definable set, we have to show that this set is dense in $X^{i}$. Let us assume that the contrary holds, that is, the set $w_{f}^{\prime}\left(X^{i}, X^{j}\right)$ of points of $X^{i}$ at which the pair $\left(X^{i}, X^{j}\right)$ is not $\left(w_{f}\right)$-regular contains a nonempty open subset of $X^{i}$, and let us obtain a contradiction. Up to replacing $X^{i}$ by a nonempty subset of $X^{i}$ and by the definability of $w_{f}^{\prime}\left(X^{i}, X^{j}\right)$, we may suppose that $w_{f}^{\prime}\left(X^{i}, X^{j}\right)$ equals $X^{i}$.

As the condition $\left(w_{f}\right)$ is invariant under differentiable transformations of $K^{n}$ with Lipschitz continuous derivative and up to replacing $X^{i}$ by a nonempty open subset 
of $X^{i}$, we may assume that $X^{i}$ is an open definable subset of $K^{m} \times\{0\}^{n-m}$. (The latter transformation exists by cell decomposition, after shrinking $X^{i}$ if necessary.) Up to replacing $X^{i}$ by a nonempty subset, we may also assume that $f_{\mid X^{i}}$ is constant, equal to 0 for simplicity. Indeed, since $w_{f}^{\prime}\left(X^{i}, X^{j}\right)$ equals $X^{i}$, we can replace $X^{i}$ by $f_{\mid X^{i}}^{-1}(a)$ with $a \in f\left(X^{i}\right)$, since the pair $\left(f_{\mid X^{i}}^{-1}(a), X^{j}\right)$ is $\left(w_{f}\right)$-regular at none of the points of $f_{\mid X^{i}}^{-1}(a)$.

Now we have two cases to consider:

Case 1. $f_{\mid X^{j}}$ is constant (in a neighborhood of $X^{i}$ ). Then condition $\left(w_{f}\right)$ is condition $(w)$. We proceed as follows.

Write $X^{i}=U \times\{0\}^{n-m}$ with $U$ open in $K^{m}$. By the cell decomposition theorem and the existence of definable choice functions and up to making $U$ smaller, there exists a definable $C^{1}$ function $\rho: U \times C \rightarrow X^{j}$ (called a $C^{1}$ wing in $X^{j}$ in [36]), where $C$ is a one-dimensional cell in $K^{\times}$with $0 \in \bar{C}$, such that $\rho(x, t)=(x, r(x, t))$ and $|r(x, t)|<|t|$, and furthermore, $w_{f}^{\prime}\left(X^{i}, X^{j}\right)$ being assumed equal to $X^{i}$, we may ask that for all $x, t$

$$
\frac{\delta\left(K^{m} \times\{0\}^{n-m}, T_{\rho(x, t)} X^{j}\right)}{|r(x, t)|} \geq|t|^{-1} .
$$

By Remark 4.2.1, one then has

$$
\frac{\left\|D_{x} r_{(x, t)}\right\|}{|r(x, t)|} \geq \frac{\delta\left(K^{m} \times\{0\}^{n-m}, T_{\rho(x, t)} X^{j}\right)}{|r(x, t)|} .
$$

By cell decomposition and up to replacing the function $(x, t) \mapsto r(x, t)$ by $(x, t) \mapsto$ $r\left(x, t^{s}\right)$ for some integer $s>0$, we may moreover assume that on $U \times C$

$$
|r(x, t)|=|a| \cdot|t|^{\ell}
$$

for some integer $\ell>0$ and some $a \in K^{\times}$. But when one applies Lemma 4.2.4 to $(x, t) \mapsto r(x, t) / t^{\ell}$, which is a bounded map, one finds a definable nonempty open subset $U^{\prime}$ of $U$, and $d \in K^{\times}$such that for $x \in U^{\prime}$ and $t \in C$ with $|t|$ small enough, $\left\|D_{x} r_{(x, t)}\right\| /|r(x, t)| \leq|d|$, a contradiction with the above two displayed inequalities.

Case 2. $f_{\mid X^{j}}$ has rank 1 .

Write $X^{i}=U \times\{0\}^{n-m}$ with $U$ open in $K^{m}$. Clearly we may suppose that $f(x, y) \neq 0$ for $(x, y) \in X^{j}, x \in U$. We further have that for each $x \in U, f(x, y)$ goes to 0 when $y$ goes to zero with $(x, y) \in X^{j}$. Hence there exists a definable choice function $f_{0}: B(0,1) \rightarrow f\left(X^{j}\right) \cup\{0\}$ such that $f_{0}(t)=0$ if and only if $t=0$ and $\left|f_{0}(t)\right|<|t|$ for nonzero $t$. Since we assume that $w_{f}^{\prime}\left(X^{i}, X^{j}\right)$ equals $X^{i}$, we may moreover assume that for each $x \in U$ and nonzero $t$ there exists $y$ satisfying $(x, y) \in X^{j},|y|<|t|, f(x, y)=f_{0}(t)$, and

$$
\frac{\delta\left(K^{m} \times\{0\}^{n-m}, T_{(x, y)} X_{f}^{j}\right)}{|y|} \geq|t|^{-1} .
$$


Up to replacing $f_{0}$ by $t \mapsto f_{0}\left(\lambda t^{s}\right)$ for some integer $s>0$ and nonzero $\lambda \in R$, we may suppose that $f_{0}$ is continuous. Hence, by the existence of definable choice functions there exists a continuous definable map $\varphi: U \times B(0,1) \rightarrow K^{n-m}$ which is $C^{1}$ on $U \times(B(0,1) \backslash\{0\})$ and such that, for all $x \in U$ and for all nonzero $t \in B(0,1)$, $\varphi(x, 0)=0,(x, \varphi(x, t)) \in X^{j}$,

$$
\frac{\delta\left(K^{m} \times\{0\}^{n-m}, T_{(x, \varphi(x, t))} X_{f}^{j}\right)}{|\varphi(x, t)|} \geq|t|^{-1}
$$

and

$$
f(x, \varphi(x, t))=f_{0}(t) .
$$

It follows by (4.2.2) that the $m$-dimensional linear space $W$ spanned by the vectors $\left(0, \ldots, 0,1,0, \ldots, 0, \partial \varphi(x, t) / \partial x_{i}\right)$ for $i=1, \ldots, m$ is a subspace of $T_{(x, \varphi(x, t))} X_{f}^{j}$. Combining this with Remark 4.2.1 and since $\left\|D_{x} \varphi_{(x, t)}\right\| \geq \delta\left(K^{m} \times\{0\}^{n-m}, W\right)$, it follows that

$$
\frac{\left\|D_{x} \varphi_{(x, t)}\right\|}{|\varphi(x, t)|} \geq \frac{\delta\left(K^{m} \times\{0\}^{n-m}, T_{(x, \varphi(x, t))} X_{f}^{j}\right)}{|\varphi(x, t)|} .
$$

By the Cell Decomposition Theorem 1.4.2, by making $U$ smaller, and up to replacing the function $(x, t) \mapsto \varphi(x, t)$ by $(x, t) \mapsto \varphi\left(x, b t^{s}\right)$ for some integer $s>0$ and some nonzero $b \in R$, we may suppose we have on $U \times B(0,1)$

$$
|\varphi(x, t)|=|a| \cdot|t|^{\ell},
$$

with $a \in K^{\times}$and some integer $\ell>0$, since $\varphi$ is continuous and $\varphi(x, t)=0$ if and only if $t=0$. Applying Lemma 4.2.4 to the bounded function $\varphi(x, t) / t^{\ell}$ yields a contradiction with (4.2.1) and (4.2.3) similarly as in case 1.

4.3. Let $X$ be a definable subset of $K^{n}$, and let $\left(X^{j}\right)_{j \in\{1, \ldots, k\}}$ be a finite, definable and analytic stratification of $X$ satisfying the frontier condition as in 4.2. Let $X^{i}$ and $X^{j}$ be strata with $X^{i} \subset \overline{X^{j}}$ and let $x_{0} \in X^{i}$. One says $\left(X^{i}, X^{j}\right)$ satisfies condition (b) at $x_{0}$ if for every sequences $x_{m} \in X^{i}, y_{m} \in X^{j}$, both converging to $x_{0}$ and such that the line $L_{m}$ containing $x_{m}$ and $y_{m}$, resp. the tangent space $T_{y_{m}} X^{j}$, both converge in the appropriate Grassmann manifold to a line $L$, resp. a subspace $T$, then $L \subset T$. Over the reals, it is well known since the seminal work of T. C. Kuo [28] (in the semi-analytic case), see also [43] (subanalytic case) and [36] (o-minimal case), that condition $(w)$ implies condition $(b)$. Note that obviously $(w)$ does not imply $(b)$ in the real differential case and that even in the real algebraic case $(b)$ does not imply $(w)$. In the present setting, we have a similar result (with a similar proof):

4.3.1 Proposition. If $\left(X^{i}, X^{j}\right)$ satisfies condition $(w)$ at $x_{0}$, it also satisfies condition (b) at $x_{0}$. 
Proof. We set $X^{i}=W$ and $X^{j}=W^{\prime}$. We may assume that $W$ is open in $K^{r} \simeq$ $K^{r} \times\{0\} \subset K^{r} \times K^{s}=K^{n}$ and that $x_{0}=0$. Denote by $p$ the linear projection $K^{n} \rightarrow K^{s}$. If condition $(b)$ is not satisfied at 0 , then, by condition $(w)$ at 0 and for some $\varepsilon>0$, one has $0 \in \bar{S} \backslash S$, with

$$
S=\left\{x \in W^{\prime} \mid \delta\left(K p(x), T_{x} W^{\prime}\right) \geq 2 \varepsilon\right\} .
$$

Use the Curve Selection Lemma 1.4.4 to find an analytic definable function $\varphi: U \subset$ $K \rightarrow S$ with $0 \in \bar{U}$ such that $\|\varphi(t)\| \leq|t|$ for all $t$ in $U$. Write $\varphi=(a, b)$ with $a: U \rightarrow K^{r}$ and $b: U \rightarrow K^{s}$. We may assume that $\left\|a^{\prime}(t)\right\|$ is bounded, that $b$ and $b^{\prime}$ do not vanish, and by analyticity that $\lim _{t \rightarrow 0}\|b(t)\| /\left\|b^{\prime}(t)\right\|=0$. Since $\delta\left(K b^{\prime}(t), K b(t)\right) \rightarrow 0$ for $t \rightarrow 0$ which holds by Lemma 1.4.5, we have $\delta\left(K b^{\prime}(t), T_{\varphi(t)} W^{\prime}\right) \geq \varepsilon$ for $t$ small enough. From the fact that $\varphi^{\prime}(t)=a^{\prime}(t)+b^{\prime}(t) \in$ $T_{\varphi(t)} W^{\prime}$, it follows that

$$
\frac{\left\|a^{\prime}(t)\right\|}{\left\|b^{\prime}(t)\right\|} \delta\left(K a^{\prime}(t), T_{\varphi(t)} W^{\prime}\right) \geq \varepsilon .
$$

Now, by condition $(w)$ at 0 , there exists $C>0$ such that

$$
\delta\left(K a^{\prime}(t), T_{\varphi(t)} W^{\prime}\right) \leq C\|b(t)\|
$$

for $t$ small enough. It follows from (4.3.1) and (4.3.2), that, for $t$ small enough,

$$
\varepsilon \leq C \frac{\|b(t)\|}{\left\|b^{\prime}(t)\right\|}\left\|a^{\prime}(t)\right\|,
$$

which contradicts the fact that $\left\|a^{\prime}(t)\right\|$ is bounded and $\lim _{t \rightarrow 0}\|b(t)\| /\left\|b^{\prime}(t)\right\|=0$.

\section{Proof of Theorem 3.6.2 and the existence of distinguished tangent $\boldsymbol{\Lambda}$-cones}

5.1. Proof of Theorem 3.6.2: a first reduction. The statement we have to prove being additive, we may cut $X$ into finitely many definable pieces. Also note that we may assume all these pieces have dimension $d$ around $x$, since pieces of dimension $<d$ contribute to zero in both sides in the equality we have to prove. Let us prove in this subsection that we may reduce to the case were $\varphi=\mathbf{1}_{X}$. Suppose that we know the result for $\varphi=\mathbf{1}_{X}$. For the general case we may assume, by additivity and linearity, that $\varphi=\left(\prod_{i=1}^{\ell} \beta_{i}\right) \cdot q^{-\alpha}$ with $\alpha$ and the $\beta_{i}$ definable functions from $X$ to $\mathbb{Z}$. Further we may assume that $\varphi \geq 0$ on $X$. Write $X$ as a possibly infinite disjoint union parameterized by the values of $\alpha$ and the $\beta_{i}$. That is,

$$
X=\bigcup_{z \in \mathbb{Z}^{\ell+1}} X_{z}, \quad \text { with } X_{z}=\left\{x \in X \mid\left(\beta_{1}, \ldots, \beta_{\ell}, \alpha\right)(x)=z\right\} .
$$


Since $\varphi$ is constant on each of the $X_{z}$, by linearity we find for each $z$

$$
\Theta_{d}\left(\varphi_{z}\right)(x)=\Theta_{d}\left(v_{x}^{\Lambda}\left(\varphi_{z}\right)\right)(0),
$$

where $\varphi_{z}$ is the product of $\varphi$ with the characteristic function of $X_{z}$. By Proposition 2.4.2 one finds

$$
\Theta_{d}(\varphi)(x)=\Theta_{d}\left(\sum_{z} \varphi_{z}\right)(x)=\sum_{z} \Theta_{d}\left(\varphi_{z}\right)(x)
$$

and similarly

$$
\begin{aligned}
\Theta_{d}\left(v_{x}^{\Lambda}(\varphi)\right)(0) & =\Theta_{d}\left(v_{x}^{\Lambda}\left(\sum_{z} \varphi_{z}\right)\right)(0)=\Theta_{d}\left(\sum_{z} v_{x}^{\Lambda}\left(\varphi_{z}\right)\right)(0) \\
& =\sum_{z} \Theta_{d}\left(v_{x}^{\Lambda}\left(\varphi_{z}\right)\right)(0)
\end{aligned}
$$

and hence $\Theta_{d}(\varphi)(x)=\Theta_{d}\left(v_{x}^{\Lambda}(\varphi)\right)(0)$ which finishes the reduction.

5.2. Proof of Theorem 3.6.2: the case $\boldsymbol{d}=\boldsymbol{n}$. In this subsection, we consider the case $d=n$. It is not difficult to see (cf. Corollary 5.3 .8 below) that the function $S C_{x}^{\Lambda}(X)$ is equal to the characteristic function of $C_{x}^{\Lambda}(X)$ almost everywhere. Hence it is enough to prove that

$$
\Theta_{d}(X)(x)=\Theta_{d}\left(C_{x}^{\Lambda}(X)\right)(0)
$$

for $\Lambda$ small enough.

The proof we shall give is quite analogous to the one of Proposition 2.1 in [32]. By Corollary 3.2.3, there exists a definable function $\alpha: \mathbf{P}^{n-1}(K) \rightarrow \mathbb{N}$ and a subgroup $\Lambda$ in $\mathcal{D}$ such that for every $\ell$ in $\mathbf{P}^{n-1}(K),\left(\pi_{x}^{X}\right)^{-1}(\ell) \cap B(x, \alpha(\ell))$ is a local $\Lambda$-cone with origin $x$ in $\left(\pi_{x}^{X}\right)^{-1}(\ell)$.

For every $n \geq 0$, we consider the $\Lambda$-cone $C_{n}(\Lambda)$ with origin $x$ generated by $X \cap B(x, n)$. Note that

$$
C_{x}^{\Lambda}(X)=\bigcap_{n} \overline{C_{n}(\Lambda)},
$$

hence, if we set

$$
W:=\bigcap_{n} C_{n}(\Lambda),
$$

we have $W \subset C_{x}^{\Lambda}(X)$. In particular, $\Theta_{d}(W)(x) \leq \Theta_{d}\left(C_{x}^{\Lambda}(X)\right)(0)$. By Proposition 2.4.2, we know that $\Theta_{d}(W)(x)=\lim _{n} \Theta_{d}\left(C_{n}(\Lambda)\right)(x)$ and $\Theta_{d}\left(C_{x}^{\Lambda}(X)\right)(0)=$ $\lim _{n} \Theta_{d}\left(\overline{C_{n}(\Lambda)}\right)(x)$. By Proposition 2.4.1, we deduce that

$$
\Theta_{d}(W)(x)=\Theta_{d}\left(C_{x}^{\Lambda}(X)\right)(0) .
$$

Since we have

$$
\Theta_{d}(X)(x)=\Theta_{d}(X \cap B(x, n))(x) \leq \Theta_{d}\left(C_{n}(\Lambda)\right)(x),
$$


we deduce that

$$
\Theta_{d}(X)(x) \leq \Theta_{d}(W)(x) .
$$

To prove the reverse inequality, let us consider for $n \geq 0$ the definable subset $W_{n}$ of all points $w$ in $W$ such that $\alpha\left(\pi_{x}(w)\right) \leq n$. By definition $W_{n} \cap B(x, n) \subset X \cap B(x, n)$, hence

$$
\Theta_{d}\left(W_{n}\right)(x)=\Theta_{d}\left(W_{n} \cap B(x, n)\right)(x) \leq \Theta_{d}(X \cap B(x, n))(x)=\Theta_{d}(X)(x) .
$$

Since, by Proposition 2.4.2 again, $\lim _{n} \Theta_{d}\left(W_{n}\right)(x)=\Theta_{d}(W)(x)$, we obtain $\Theta_{d}(W)(x) \leq \Theta_{d}(X)(x)$, as required.

5.3. Graphs. The main technical result in this subsection is Proposition 5.3.7, which will be used in subSection 5.5 to conclude the proof of Theorem 3.6.2 and in subSection 5.6 to prove the existence of distinguished tangent $\Lambda$-cones.

Fix two integers $0<d \leq m$. Let $U$ be an open definable subset of $K^{d}$ and let $\varphi$ be a definable mapping $U \rightarrow K^{m-d}$. The graph $\Gamma=\Gamma(\varphi)$ of $\varphi$ is a definable subset of $K^{m}$. Fix a point $u$ in the closure $\bar{U}$ of $U$ and $\Lambda$ adapted to $(U, u)$. We assume that $\lim _{x \rightarrow u} \varphi(x)=v$, by Corollary 3.4.2. We set $w:=(u, v)$. The projection to the first $d$ coordinates $K^{m} \rightarrow K^{d}$ induces a function

$$
\vartheta: \mathscr{D}(\Gamma, w, \Lambda) \longrightarrow \mathscr{D}(U, u, \Lambda) .
$$

Note that $\vartheta$ is an isomorphism with inverse given by

$$
\vartheta^{-1}:(z, \lambda) \longmapsto\left(z, \lambda^{-1}(\varphi(u+\lambda z)-v), \lambda\right) .
$$

By Corollary 3.2.3, there exists a definable function $\alpha: \mathbf{P}^{d-1}(K) \rightarrow \mathbb{N} \cup\{\infty\}$ such that for every $\ell$ in $\mathbf{P}^{d-1}(K),\left(\pi_{u}^{U}\right)^{-1}(\ell) \cap B(u, \alpha(\ell))$ is a local $\Lambda$-cone with origin $u$ in $\left(\pi_{u}^{U}\right)^{-1}(\ell)$, with the convention that $\alpha(\ell)=\infty$ if and only if $\ell$ is such that $\left(\pi_{u}^{U}\right)^{-1}(\ell) \cap U=\emptyset$. Note that being definable, the function $\alpha$ is continuous on a dense definable open subset $\Omega_{0}$ in $\mathbf{P}^{d-1}(K)$, where dense means that the complement of $\Omega_{0}$ has strictly smaller dimension. Let $\Omega_{1}$ be the definable subset of $\Omega_{0}$ consisting of the $\ell$ such that for all neighborhoods $\mathcal{V}$ of $u$ in $K^{d}$, the sets $\left(\pi_{u}^{U}\right)^{-1}(\ell) \cap \mathcal{V}$ are nonempty.

5.3.1 Lemma. Suppose that $C_{u}^{\Lambda}(U)$ is of maximal dimension. Then $\Omega_{1}$ contains a nonempty open subset of $\mathbf{P}^{d-1}(K)$.

Proof. Let $\Omega_{1}^{c}$ be the complement of $\Omega_{1}$ in $\mathbf{P}^{d-1}(K)$. Clearly $\Omega_{1}^{c}$ is definable. It is enough to derive a contradiction out of the assumption that $\Omega_{1}^{c}$ is dense. Suppose thus that $\Omega_{1}^{c}$ is dense in $\mathbf{P}^{d-1}(K)$. By the definability and density of $\Omega_{1}^{c}$ and of $\Omega_{0}$ in $\mathbf{P}^{d-1}(K)$, it follows that $\Omega_{1}^{c} \cap \Omega_{0}$ is dense. Take $\ell$ in $\Omega_{1}^{c} \cap \Omega_{0}$. By the definition of the tangent cone, one has that $\left(\pi_{u}^{U}\right)^{-1}(\ell) \cap C_{u}^{\Lambda}(U)=\emptyset$. Since $\left(\pi_{u}^{U}\right)^{-1}\left(\Omega_{1}^{c} \cap \Omega_{0}\right)$ is 
dense and definable in $K^{d}$, it follows that $C_{u}^{\Lambda}(U)$ is contained in a definable subset of dimension $<d$, a contradiction with $C_{u}^{\Lambda}(U)$ being of maximal dimension, that is, of dimension $d$.

For any definable subset $O$ of $\mathbf{P}^{d-1}(K)$, consider the definable subset

$$
C_{u}^{\Lambda, O}(U):=\left(\pi_{u}^{U}\right)^{-1}(O) \cap C_{u}^{\Lambda}(U)
$$

of $C_{u}^{\Lambda}(U)$.

5.3.2 Lemma. Suppose that $C_{u}^{\Lambda}(U)$ is of maximal dimension. Let $O$ be dense open in $\Omega_{1}$. Then the set $C_{u}^{\Lambda, O}(U)$ also has maximal dimension and is dense open in $C_{u}^{\Lambda}(U)$.

Proof. The fact that $C_{u}^{\Lambda, O}(U)$ is open in $C_{u}^{\Lambda}(U)$ follows from general topology. We only have to prove that $C_{u}^{\Lambda, O}(U)$ is dense in $C_{u}^{\Lambda}(U)$. As we have noticed in the proof of Lemma 5.3.1, for every $\ell$ in $\Omega_{1}^{c} \cap \Omega_{0}$,

$$
\left(\pi_{u}^{U}\right)^{-1}(\ell) \cap C_{u}^{\Lambda}(U)=\emptyset .
$$

Since moreover the sets $\left(\pi_{u}^{U}\right)^{-1}\left(\Omega_{1} \backslash O\right)$ and $\left(\pi_{u}^{U}\right)^{-1}\left(\Omega_{1}^{c} \backslash \Omega_{0}\right)$ have dimension $<d$, the lemma follows.

The next lemma ensures in particular that there exist definable sets $\Omega=O$ as in Lemma 5.3.2 such that moreover for all $z$ in $C_{u}^{\Lambda, \Omega}(U)$ and all small enough $\lambda$ in $\Lambda$ one has that $u+\lambda z$ lies in $U$.

5.3.3 Lemma. Suppose that $C_{u}^{\Lambda}(U)$ is of maximal dimension. Then there is a dense open definable subset $\Omega$ of $\Omega_{1}$ such that for all $z$ in $C_{u}^{\Lambda, \Omega}(U)$ of direction $\ell$ and for all small enough $\lambda$ in $\Lambda$ one has $u+\lambda z \in \overline{\left(\pi_{u}^{U}\right)^{-1}(\ell)}$. Here, small enough can be taken to mean that $\operatorname{ord}(\lambda z) \geq \alpha(\ell)$, where $\alpha$ is as in the beginning of Section 5.3.

Proof. We assume $u=0$ for simplicity. For any $\ell \in \Omega$, any $z \in \ell \cap C_{u}^{\Lambda}(U)$ and any $\lambda \in \Lambda$, one has $\lambda z \in C_{u}^{\Lambda}(U)$. Hence what remains to be proved is a consequence of the inclusion $\subset$ in the equality of the following claim.

5.3.4 Claim. For almost all $\ell$ in $\Omega_{1}$ and with $u=0$, one has the following equality of local $\Lambda$-cones

$$
C_{u}^{\Lambda}(U) \cap \ell \cap B(0, \alpha(\ell))=\left(\overline{\left(\pi_{u}^{U}\right)^{-1}(\ell)} \cap B(0, \alpha(\ell))\right.
$$


Proof of the claim. Since $\ell \in \Omega_{1},\left(\pi_{u}^{U}\right)^{-1}(\ell) \cap B(0, \alpha(\ell))$ is a local $\Lambda$-cone with origin 0 , and by Remark 3.3.1, we have

$$
\begin{aligned}
\overline{\left(\pi_{u}^{U}\right)^{-1}(\ell)} \cap B(0, \alpha(\ell)) & =C_{u}^{\Lambda}\left(\left(\pi_{u}^{U}\right)^{-1}(\ell)\right) \cap B(0, \alpha(\ell)) \\
& \subset C_{u}^{\Lambda}(U) \cap \ell \cap B(0, \alpha(\ell)) .
\end{aligned}
$$

The inclusion $\supset$ is thus clear for all $\ell \in \Omega$. We prove the inclusion $\subset$ in the claim, for almost all $\ell$. This follows from cell decomposition. Let $X \subset\left(K^{d} \backslash\{0\}\right) \times K$ be the definable set

$$
\left\{(x, t) \in\left(K^{d} \backslash\{0\}\right) \times K \mid u+t \cdot x \in U\right\},
$$

parametrizing all $\ell \cap U$ for all lines $\ell$ through $u$. Then $X$ is a finite union of cells by Theorem 1.4.2. For each $x \in K^{d} \backslash\{0\}$ write $X_{x}$ for the fiber above $x$ under the projection $X \rightarrow K^{d}$. For each $x$, either 0 lies in the interior of $X_{x}$, either 0 lies in the boundary $\partial X_{x}$ of $X_{x}$ or 0 lies outside the closure of $X_{x}$, where $\partial X_{x}$ is the closure of $X$ minus the interior of $X$. In the case that 0 lies in the interior of $X_{x}$, one has that $\left(\pi_{u}^{U}\right)^{-1}(\ell) \cap B(u, \alpha(\ell))=B(u, \alpha(\ell))$ hence the inclusion $\subset$ is evident.

The inclusion $\subset$ holds, up to a set of direction $\ell \in \mathbf{P}^{d-1}(K)$ of dimension $<d-1$, for all those $x$ such that 0 lies in $\partial X_{x}$ by the almost everywhere continuity of the functions in $x$ appearing in the descriptions of the cells having 0 in their boundary. The case that 0 lies outside the closure of $X_{x}$ needs not to be considered since we suppose $\ell \in \Omega_{1}$.

5.3.5 Corollary. Let $d>0$, let $U$ be a definable nonempty open subset of $K^{d}$ and let $\Lambda$ given by Corollary 3.2.3. Then for all $u \in \bar{U}$, with $C_{u}^{\Lambda}(U)$ of maximal dimension $d, C_{u}^{\Lambda}(U)$ is a distinguished $\Lambda$-tangent cone at $u$ for $U$, that is, for all $\Lambda^{\prime} \in \mathcal{D}$, $\Lambda^{\prime} \subset \Lambda$ implies $C_{u}^{\Lambda^{\prime}}(U)=C_{u}^{\Lambda}(U)$.

Proof. As usual we assume $u=0$. Let $\Lambda$ as given by Corollary 3.2.3 and $\Lambda^{\prime} \in \mathscr{D}$, $\Lambda^{\prime} \subset \Lambda$. We show that $C_{u}^{\Lambda}(U) \subset C_{u}^{\Lambda^{\prime}}(U)$. Let $z \in C_{u}^{\Lambda}(U)$, denote by $\ell$ its direction and assume that $\ell \in \Omega$, with $\Omega \subset \Omega_{1}$ as in Lemma 5.3.3. By Lemma 5.3.3 we then have $z \in C_{u}^{\Lambda}(\overline{U \cap \ell})=C_{u}^{\Lambda}(U \cap \ell)$. But since $U \cap \ell \cap B(0, \alpha(\ell))$ is a local $\Lambda$-cone with origin $u$, by Remark 3.3.1, we get

$$
z \in C_{u}^{\Lambda}(U \cap \ell)=C_{u}^{\Lambda^{\prime}}(U \cap \ell) \subset C_{u}^{\Lambda^{\prime}}(U)
$$

Now since we showed $C_{u}^{\Lambda, \Omega}(U)=C_{u}^{\Lambda^{\prime}, \Omega}(U)$, we have $\overline{C_{u}^{\Lambda, \Omega}(U)}=\overline{C_{u}^{\Lambda^{\prime}, \Omega}(U)}$. But by Lemma 5.3.2 we obtain $\overline{C_{u}^{\Lambda, \Omega}(U)}=C_{u}^{\Lambda}(U)$. We finally remark that one also has $\overline{C_{u}^{\Lambda^{\prime}, \Omega}(U)}=C_{u}^{\Lambda^{\prime}}(U)$, with the same proof as in Lemmas 5.3.1 and 5.3.2, since any adapted (to $U$ ) $\Lambda^{\prime}$-cone may be chosen in those lemmas. 
Proposition 5.3.7 below can be seen as an analogue of Proposition 3.6 of [32] and has for consequence the existence of distinguished $\Lambda$-tangent cones for general definable sets and the $p$-adic analogue of Thie's formula. As usual, the main point in the $p$-adic case is to overcome the lack of connectedness and deal with all its negative consequences such as the lack of strong enough mean value theorems and so forth. To go through these difficulties we essentially use the following result, the main result of [5], which is the $p$-adic analogue of the existence of the so-called $L$-decompositions of real subanalytic sets, obtained in [29], and which will be used in the proof of Proposition 5.3.7 (c).

5.3.6 Theorem. Let $\varepsilon>0$ and let $\varphi: X \subset K^{n} \rightarrow K^{m}$ be a locally $\varepsilon$-Lipschitz definable mapping. Then there exist $C>0$ and a finite definable partition of $X$ into parts $X_{1}, \ldots, X_{k}$ such that the restriction of $\varphi$ to each $X_{i}$ is globally $C$-Lipschitz.

We will also use Theorem 4.2.5 in the proof of Proposition 5.3.7 in the same way that Lemma 3.7 of [32] is used in the proof of Proposition 3.6 of [32]. Where Theorem 4.2.5 gives the existence of $\left(w_{f}\right)$-regular (and consequently $\left(a_{f}\right),(b)$, and $(w)$-regular) stratifications for a function $f$ in the definable $p$-adic setting, we will only use the genericity of the condition $\left(a_{f}\right)$ in the $p$-adic definable case to prove Proposition 5.3.7.

5.3.7 Proposition. Let $\varepsilon$ be a positive real number with $\varepsilon \leq 1$. Let $U$ be an open definable subset of $K^{d}$ and let $\varphi$ be a definable mapping $U \rightarrow K^{m-d}$. Fix a point $u$ in $\bar{U}$, a subgroup $\Lambda$ adapted to $(U, u)$, choose $\Omega$ sufficiently small and as in Lemma 5.3.3, and let $C_{u}^{\Lambda, \Omega}(U)$ be as in (5.3.2). Assume that $\varphi$ is $\varepsilon$-analytic and that $\lim _{x \rightarrow u} \varphi(x)=v$ by Corollary 3.4.2.

Suppose that $C_{u}^{\Lambda}(U)$ has maximal dimension. Then, possibly after partitioning $U$ into finitely many open subsets, replacing $U$ successively by each one of these smaller open subsets, in such a way that $\varphi$ is globally $C$-Lipschitz on $U$ by Theorem 5.3.6 and neglecting those $U$ such that $C_{u}^{\Lambda}(U)$ has lower dimension, the following hold

(a) For $z$ in $C_{u}^{\Lambda, \Omega}(U)$ such that $u+\lambda z \in U$, for all small enough $\lambda \in \Lambda$ (see Lemma 5.3.3), the limit

$$
\psi(z):=\lim _{\substack{\lambda \rightarrow 0 \\ \lambda \in \Lambda}} \lambda^{-1}(\varphi(u+\lambda z)-v)
$$

exists in $K^{m-d}$, yielding a definable function $\psi: C_{u}^{\Lambda, \Omega}(U) \rightarrow K^{m-d}$.

(b) The function $\psi$ is locally $\varepsilon$-Lipschitz.

(c) The graph of $\psi$ is dense in $C_{w}^{\Lambda}(\Gamma(\varphi))$.

Proof. We first prove (a). Choose $z$ in $C_{u}^{\Lambda, \Omega}(U)$ such that $u+\lambda z \in U$, for all small enough $\lambda \in \Lambda$. We can evaluate $\varphi$ at $u+\lambda z$ for small enough $\lambda$ in $\Lambda$. After 
partitioning $U$ into finitely many open subsets and successively replacing $U$ by each one of these smaller open subsets, Lemma 1.5.5 implies that when $\lambda \rightarrow 0, \lambda \in \Lambda$, either $\lambda^{-1}(\varphi(u+\lambda z)-v)$ has a limit $\psi(z)$ or its norm goes to $\infty$. Applying the Curve Selection Lemma 1.4.4 to the point $(u, v)$ and the set

$$
\left\{(u+\lambda z, \varphi(u+\lambda z)) \in K^{m} \mid \lambda \in \Lambda\right\},
$$

it follows from Lemma 1.4 .5 and $\varepsilon$-analyticity that the limit $\psi(z)$ exists.

Now assume that $z \in C_{u}^{\Lambda, \Omega}(U)$ is such that $u+\lambda z \in \overline{U \cap \ell}$, where $\ell$ is the (direction of the) line going through $u$ and $z$. By Lemma 5.3.3, for all $\varepsilon>0$ there exist $z^{\prime}, z^{\prime \prime} \in C_{u}^{\Lambda, \Omega}(U)$ such that $u+\lambda z^{\prime} \in U, u+\lambda z^{\prime \prime} \in U$ for all $\lambda \in \Lambda$ and $\left|z-z^{\prime}\right| \leq \varepsilon$ and $\left|z-z^{\prime \prime}\right| \leq \varepsilon$. Then we have

$$
\left|\lambda^{-1}\left[\left(\varphi\left(u+\lambda z^{\prime}\right)-v\right)-\left(\varphi\left(u+\lambda z^{\prime \prime}\right)-v\right)\right]\right| \leq C\left|\lambda^{-1} \lambda\left(z^{\prime}-z^{\prime \prime}\right)\right| \leq C \cdot \varepsilon .
$$

This shows that one can define $\psi$ on $C_{u}^{\Lambda, \Omega}$ by

$$
\psi(z)=\lim _{z^{\prime} \rightarrow z} \lim _{\lambda \rightarrow 0} \lambda^{-1}\left(\varphi\left(u+\lambda z^{\prime}\right)-v\right) .
$$

Let us now prove (b). We first notice that, after suitable finite partition of $U$ and neglecting those $U$ such that $C_{u}^{\Lambda}(U)$ has lower dimension, we may suppose that the function $\psi$ is analytic on $C_{u}^{\Lambda, \Omega}(U)$. To prove that $\psi$ is $\varepsilon$-analytic on $C_{u}^{\Lambda, \Omega}(U)$, we show that the tangent space $T_{x} \Gamma(\psi)$ at a point $x$ of the graph $\Gamma(\psi)$ of $\psi$, for $x$ in a dense set of $\Gamma(\psi)$, is contained in $C_{\varepsilon}=\left\{(a, b) \in K^{d} \times K^{m-d}|| b|\leq \varepsilon| a \mid\right\}$. Since, by (5.3.3), $\Gamma(\psi) \subset C_{w}^{\Lambda}(\Gamma(\varphi))$ and since $\operatorname{dim}(\Gamma(\psi))=\operatorname{dim}\left(C_{w}^{\Lambda}(\Gamma(\varphi))\right)$, it is enough to prove that at a generic point $x$ of $C_{w}^{\Lambda}(\Gamma(\varphi))$ one has $T_{x} C_{w}^{\Lambda}(\Gamma(\varphi)) \subset C_{\varepsilon}$. For this we consider the deformation $h: \overline{\mathscr{D}(\Gamma(\varphi), w, \Lambda)} \rightarrow K$ to $C_{w}^{\Lambda}(\Gamma(\varphi))$ defined in section 3.5. The fiber $h^{-1}(0)$ is identified with $C_{w}^{\Lambda}(\Gamma(\varphi))$ and for $\lambda \in \Lambda$

$$
h^{-1}(\lambda)=\left\{(z, \lambda) \in K^{m} \times \Lambda \mid w+\lambda z \in \Gamma(\varphi)\right\}
$$

is identified with

$$
\left\{z \in K^{m} \mid w+\lambda z \in \Gamma(\varphi)\right\} .
$$

Since $\varphi$ is $\varepsilon$-analytic, for any $\lambda \in \Lambda$ and any $y \in h^{-1}(\lambda)$, one has $T_{y} h^{-1}(\lambda) \subset C_{\varepsilon}$. Let us show at $x$ a generic point of $C_{w}^{\Lambda}(\Gamma(\varphi)), T_{x} C_{w}^{\Lambda}(\Gamma(\varphi))$ is a limit of tangents $T_{y_{n}} h^{-1}\left(\lambda_{n}\right)$. But this is exactly the genericity in $h^{-1}(0)$ of the condition $\left(a_{h}\right)$, which is given by Theorem 4.2.5.

We now prove (c). Let $z \in C_{w}^{\Lambda}(\Gamma(\varphi))$ and $\left(\lambda_{n}\right)_{n \in \mathbb{N}} \in \Lambda,\left(w_{n}\right)_{n \in \mathbb{N}} \in \Gamma(\varphi)$ be two sequences such that $w_{n} \rightarrow w$ and $\lambda_{n}\left(w_{n}-w\right) \rightarrow z$. Denoting by $\pi$ the projection from $\Gamma$ to $U$ and $u_{n}=\pi\left(w_{n}\right)$, the sequence $\left(u_{n}\right)_{n \in \mathbb{N}}$ of points of $U$ going to $u$ is such that $\lim _{n \rightarrow \infty} \lambda_{n}\left(u_{n}-u\right)=\pi(z):=a \in C_{u}^{\Lambda}(U)$. Now fix $\varepsilon>0$ and $a^{\prime} \in C_{u}^{\Lambda, \Omega}(U)$ with $\left|a-a^{\prime}\right| \leq \varepsilon$. Then $u+\lambda a^{\prime} \in U$ for all small enough $\lambda \in \Lambda$ by Lemma 5.3.3. Then we may suppose, by invoking Theorem 5.3.6, that

$$
\left.\left|\lambda_{n}\left(\varphi\left(u_{n}\right)-v\right)-\lambda_{n}\left(\varphi\left(\lambda_{n}^{-1} a^{\prime}+u\right)-v\right)\right| \leq C \mid \lambda_{n}\left(u_{n}-u\right)-a^{\prime}\right) \mid .
$$


This gives

$$
\lim _{n \rightarrow \infty}\left|\lambda_{n}\left(w_{n}-w\right)-\lambda_{n}\left(\lambda_{n}^{-1} a^{\prime}, \varphi\left(\lambda_{n}^{-1} a^{\prime}+u\right)\right)\right| \leq \max (C, 1) \cdot \varepsilon,
$$

and, finally

$$
\left|z-\left(a^{\prime}, \psi\left(a^{\prime}\right)\right)\right| \leq \max (C, 1) \cdot \varepsilon,
$$

showing that the graph of $\psi$ is dense in $C_{w}^{\Lambda}(\Gamma(\varphi))$.

5.3.8 Corollary. Under the hypotheses and with the notation of Proposition 5.3.7, assume moreover that $\varepsilon \leq 1$. Write $z$ for variables running over $K^{d}$ and $y$ for variables running over $K^{m-d}$. Then, for almost all $(z, y) \in C_{w}^{\Lambda}(\Gamma(\varphi))$, one has that $S C_{w}^{\Lambda}(\Gamma(\varphi))(z, y)=1=S C_{u}^{\Lambda}(U)(z)$, and

$$
\Theta_{d}\left(S C_{w}^{\Lambda}(\Gamma(\varphi))\right)(0)=\Theta_{d}\left(C_{w}^{\Lambda}(\Gamma(\varphi))\right)(0)=\Theta_{d}(U)(u)=\Theta_{d}(\Gamma(\varphi))(w) .
$$

Proof. We first prove that, for almost all $z \in C_{u}^{\Lambda}(U)$, one has that $1=S C_{u}^{\Lambda}(U)(z)$. Let $\Omega$ and $\alpha$ be as in Lemma 5.3.3. By Lemma 5.3.3, for $z \in C_{u}^{\Lambda, \Omega}(U)$, there exist an open ball $B$ contained in $C_{u}^{\Lambda, \Omega}(U)$ and containing $z$, and a ball $B_{1} \subset K$ around 0 such that

$$
\mathcal{D}(U, u, \Lambda) \cap\left(B \times B_{1}\right)=B \times\left(\Lambda \cap B_{1}\right) .
$$

Hence we can calculate

$$
\begin{aligned}
S C_{u}^{\Lambda}(U)(z) & =[K: \Lambda] \Theta_{d+1}(\mathscr{D}(U, u, \Lambda))(z, 0) \\
& =[K: \Lambda] \Theta_{d+1}(B \times \Lambda)(z, 0)=\Theta_{d}(B)(z)
\end{aligned}
$$

which equals 1 since $z \in B$.

Next we prove that $S C_{w}^{\Lambda}(\Gamma(\varphi))(z, y)=1$ for almost all $(z, y) \in C_{w}^{\Lambda}(\Gamma(\varphi))$. For this purpose, define

$$
\mathscr{D}^{\prime}:=\{(z, y, \lambda) \in \mathscr{D}(\Gamma(\varphi), w, \Lambda)|| y|\leq| z \mid\},
$$

and consider the natural projection

$$
p: \mathscr{D}^{\prime} \rightarrow \mathscr{D}(U, u, \Lambda), \quad(z, y, \lambda) \mapsto(z, \lambda),
$$

which is in fact injective. Write $U^{\prime}$ for the image of $p$. By Proposition 3.4.1 and Lemma 1.4.4, one finds for all $(z, y) \in K^{m}$ that

$$
\Theta_{d+1}\left(\mathcal{D}^{\prime}\right)(z, y, 0)=\Theta_{d+1}(\mathcal{D}(\Gamma(\varphi), w, \Lambda))(z, y, 0)
$$

and for almost all $z \in K^{d}$ that

$$
\Theta_{d+1}\left(U^{\prime}\right)(z, 0)=\Theta_{d+1}(\mathscr{D}(U, u, \Lambda))(z, 0) .
$$


Since for all $(z, y, \lambda) \in \mathscr{D}^{\prime}$ one has $|(z, y, \lambda)|=|(z, \lambda)|$, by the bijectivity of $p: \mathscr{D}^{\prime} \rightarrow U^{\prime}$, and by definition of $\Theta_{d+1}$, one finds

$$
\Theta_{d+1}\left(\mathscr{D}^{\prime}\right)(z, y, 0)=\Theta_{d+1}\left(U^{\prime}\right)(z, 0) .
$$

This shows that $S C_{w}^{\Lambda}(\Gamma(\varphi))(z, y)=1$ for almost all $(z, y) \in C_{w}^{\Lambda}(\Gamma(\varphi))$. It also follows that

$$
\Theta_{d}\left(S C_{w}^{\Lambda}(\Gamma(\varphi))\right)(0)=\Theta_{d}\left(C_{w}^{\Lambda}(\Gamma(\varphi))\right)(0) .
$$

We proceed with similar arguments to show the remaining equalities. Assume from now on until the end of the proof, for simplicity, that $w=0$. By Proposition 5.3.7(c) one has $\Theta_{d}(\Gamma(\psi))(0)=\Theta_{d}\left(C_{w}^{\Lambda}(\Gamma(\varphi))(0)\right.$. By Propositions 3.4.1 and 5.3.7 and since $\varepsilon \leq 1$, the map $z \mapsto(z, \psi(z))$ defined for $z$ in $C_{u}^{\Lambda, \Omega}(U)$ preserves the norm in the sense that $|z|=|(z, \psi(z))|$ (recall that one uses the sup-norm for tuples in an ultrametric setting). Hence, by the definition of $\Theta_{d}$, by Lemma 5.3.2 and by Section 5.2, one has $\Theta_{d}(\Gamma(\psi))(0)=\Theta_{d}\left(C_{u}^{\Lambda, \Omega}(U)\right)(0)=$ $\Theta_{d}\left(C_{u}^{\Lambda}(U)\right)(0)=\Theta_{d}(U)(0)$. Combining the obtained series of equalities yields $\Theta_{d}\left(S C_{w}^{\Lambda}(\Gamma(\varphi))\right)(0)=\Theta_{d}(U)(0)$.

Finally we prove that this value also equals $\Theta_{d}(\Gamma(\varphi))(0)$, by again a similar argument. Define $U^{\prime \prime}:=\{z \in U|| \varphi(z)|\leq| z \mid\}$. Then, by Lemma 3.4.1 and its proof based on Lemma 1.4.4, we find $C_{u}^{\Lambda}(U)=C_{u}^{\Lambda}\left(U^{\prime \prime}\right)$. Hence, $\Theta\left(C_{u}^{\Lambda}(U)\right)(0)=$ $\Theta_{d}\left(C_{u}^{\Lambda}\left(U^{\prime \prime}\right)\right)(0)$ which also equals $\Theta_{d}\left(U^{\prime \prime}\right)(0)$ by Section 5.2. Since on $U^{\prime \prime}$ the map $z \mapsto(z, \varphi(z))$ preserves the norm in the sense that $|z|=|(z, \varphi(z))|$ we find by the definition of $\Theta_{d}$ that $\Theta_{d}\left(U^{\prime \prime}\right)(u)=\Theta_{d}\left(\Gamma\left(\varphi_{\mid U^{\prime \prime}}\right)\right)(w)=\Theta_{d}(\Gamma(\varphi))(w)$ which finishes the proof.

5.4. An alternative view on cones with multiplicities.. Let $X \subset K^{n}$ be definable and of dimension $d$. It follows from Proposition 5.3.7 and its Corollary 5.3.8 that there is a finite definable partition of $X$ into parts $X_{j}$ which are graphs of $\varepsilon$-analytic Lipschitz functions on open subsets $U_{j}$ such that, for small enough $\Lambda, \Theta_{d}\left(X_{j}\right)(0)=$ $\Theta_{d}\left(C_{0}^{\Lambda}\left(X_{j}\right)\right)(0)$ for each $j$. It follows by additivity that

$$
\sum_{j} \Theta_{d}\left(C_{0}^{\Lambda}\left(X_{j}\right)\right)=\sum_{j} \Theta_{d}\left(X_{j}\right)=\Theta_{d}(X) .
$$

This common value can of course be different from $\Theta_{d}\left(C_{0}^{\Lambda}(X)\right)$ since $X_{j}$ and $X_{k}$ may have tangent cones which coincide on a part of dimension $d$ for different $j, k$, that is, there might be overlap in the union $C_{0}^{\Lambda}(X)=\bigcup_{j} C_{0}^{\Lambda}\left(X_{j}\right)$. Let us decompose $C_{0}^{\Lambda}(X)$ into parts $C_{k}, k \geq 1$, with the property that a line $\ell \subset C_{k}$ (through the origin) belongs to $C_{0}^{\Lambda}\left(X_{j}\right)$ for exactly $k$ different $j$. (Note that such decomposition is in general not unique.) Let us then define the function $C M_{0}^{\Lambda}(X)$ on $C_{0}^{\Lambda}(X)$, up to definable subsets of $C_{0}^{\Lambda}(X)$ of dimension $<d$, by

$$
C M_{0}^{\Lambda}(X)=\sum_{k} k \cdot \mathbf{1}_{C_{k}} .
$$


Any other such decomposition of $X$ into parts $X_{j}$ will yield the same function $C M_{0}^{\Lambda}(X)$ up to a definable subset of $C_{0}^{\Lambda}(X)$ of dimension $<d$, as can be seen by taking common refinements and by general dimension theory of definable sets. Clearly $\Theta_{d}\left(C M_{0}^{\Lambda}(X)\right)=\sum_{j} \Theta_{d}\left(X_{j}\right)=\Theta_{d}(X)$. Moreover, by additivity of $S C$ and by Corollary 5.3.8, for all $z \in C_{0}^{\Lambda}(X)$ up to a definable set of dimension $<d$

$$
S C_{0}^{\Lambda}(X)(z)=C M_{0}^{\Lambda}(X)(z) .
$$

In particular it follows that $S C_{0}^{\Lambda}(X)(z)$ is a nonnegative integer for all $z \in C_{0}^{\Lambda}(X)$ up to a definable set of dimension $<d$.

5.5. End of proof of Theorem 3.6.2. We consider a definable subset $X$ of dimension $d$ in $K^{n}$ and a point $x$ of $K^{n}$. We may assume $x$ lies in the closure of $X$. Let us fix $0<\varepsilon \leq 1$. By Proposition 1.5.3 there exists a decomposition

$$
X=\bigcup_{1 \leq i \leq N(\varepsilon)} \gamma_{i}\left(\Gamma_{i}(\varepsilon)\right) \cup Y
$$

with $Y$ a definable subset of $X$ of dimension $<d$, definable open subsets $U_{i}(\varepsilon)$ of $K^{d}$, for $1 \leq i \leq N(\varepsilon)$, definable analytic functions $\varphi_{i}(\varepsilon): U_{i}(\varepsilon) \rightarrow K^{m-d}$ whose graphs $\Gamma_{i}(\varepsilon)$ are all $\varepsilon$-analytic, and elements $\gamma_{1}, \ldots, \gamma_{N(\varepsilon)}$ in $\mathrm{GL}_{m}(R)$ such that the sets $\gamma_{i}\left(\Gamma_{i}(\varepsilon)\right)$ are all disjoint and contained in $X$. We denote by $u_{i}$ the image of $\gamma_{i}^{-1}(x)$ under the projection to $K^{d}$ and we fix $\Lambda$ adapted to $(X, x)$ and to $\left(U_{i}(\varepsilon), u_{i}\right)$ for every $1 \leq i \leq N(\varepsilon)$. By linearity and since the $\gamma_{i}$ 's are isometries, we have then

$$
\Theta_{d}(X)(x)=\sum_{1 \leq i \leq N(\varepsilon)} \Theta_{d}\left(\Gamma_{i}(\varepsilon)\right)\left(\gamma_{i}^{-1}(x)\right)
$$

and

$$
\Theta_{d}\left(S C_{x}^{\Lambda}(X)\right)(0)=\sum_{1 \leq i \leq N(\varepsilon)} \Theta_{d}\left(S C_{\gamma_{i}^{-1}(x)}^{\Lambda}\left(\Gamma_{i}(\varepsilon)\right)\right)(0),
$$

and the result follows from Corollary 5.3.8.

5.6. Existence of distinguished tangent $\boldsymbol{\Lambda}$-cones. We deduce from Corollary 5.3 .5 and Proposition 5.3.7 the existence of distinguished tangent $\Lambda$-cones.

5.6.1 Theorem. Let $X$ be a definable subset of $K^{n}$. Then there exists $\Lambda \in \mathcal{D}$ such that for any $x \in \bar{X}, C_{x}^{\Lambda}(X)$ is a distinguished $\Lambda$-cone, that is to say $\Lambda^{\prime} \subset \Lambda$ implies $C_{x}^{\Lambda^{\prime}}(X)=C_{x}^{\Lambda}(X)$.

Proof. We will work by induction on the dimension $d$ of $X$, where for $d=0$ the statement is trivial. We may work up to a finite partition of $X$ into definable pieces $X_{k}$ with distinguished $\Lambda_{k}$-cones $C_{x}^{\Lambda_{k}}\left(X_{k}\right)$ for all $x$ and for some $\Lambda_{k}$, since one 
can put $\Lambda:=\bigcap_{k} \Lambda_{k}$ and then $C_{x}^{\Lambda}(X)=\bigcup_{k} C_{x}^{\Lambda}\left(X_{k}\right)$ implies that $C_{x}^{\Lambda}(X)$ is a distinguished $\Lambda$-cone for all $x$. Up to a finite partition using Proposition 1.5.3 and Theorem 5.3.6, we may suppose that $X$ is the graph of some definable $C$-Lipschitz and $\varepsilon$-analytic map $\varphi: U \rightarrow K^{n-d}$, where $U$ is a definable open subset of $K^{d}$ and $d$ is the dimension of $X$.

Fix $x \in \bar{X}$ and write $u \in \bar{U}$ for the projection of $x$ in $K^{d}$. We will construct a distinguished $\Lambda$ for this fixed $x$, with the extra property that in the construction one could as well take $x$ as a parameter running over $K^{n}$ and consider the analogue of the set-up in families parameterized by $x$, and then only finitely many $\Lambda$ will come up. Taking the intersection of these finitely many $\Lambda$ as above then finishes the proof.

First suppose that $\varphi$ falls under the conditions of Proposition 5.3.7, that is, $C_{u}^{\Lambda}(U)$ has maximal dimension $d$ for some $\Lambda \in \mathscr{D}$ which is adapted to $U$. We know from Corollary 5.3.5 that $\Lambda$ is distinguished for $U$, meaning that for $\Lambda^{\prime} \subset \Lambda$ one has

$$
C_{u}^{\Lambda^{\prime}}(U)=C_{u}^{\Lambda}(U)
$$

Fix $\Lambda^{\prime} \subset \Lambda$ and consider

$$
\psi: C_{u}^{\Lambda, \Omega}(U) \rightarrow K^{n-d}
$$

and

$$
\psi^{\prime}: C_{u}^{\Lambda^{\prime}, \Omega^{\prime}}(U) \rightarrow K^{n-d}
$$

(the notation being coherent with Proposition 5.3.7). We may suppose that $\Omega=\Omega^{\prime}$. But then $C_{u}^{\Lambda, \Omega}(U)=C_{u}^{\Lambda^{\prime}, \Omega^{\prime}}(U)$ by Equation (5.6.1), and, for any $z$ in this set, we have $\psi(z)=\psi^{\prime}(z)$ by Proposition 5.3.7 (a). Hence, $\psi$ and $\psi^{\prime}$ are the same function. Taking the closures of the graph of this function, Proposition 5.3.7 (c) now yields that $C_{x}^{\Lambda^{\prime}}(X)=C_{x}^{\Lambda}(X)$ and we are done in this case.

Let us finally consider the case that $C_{x}^{\Lambda}(X)$ has dimension $<d$ for some $\Lambda$ (which happens if and only if $C_{u}^{\Lambda}(U)$ has dimension $<d$ ). We will construct a definable $Y \subset X$ such that $\operatorname{dim}(Y)=\operatorname{dim}\left(C_{x}^{\Lambda}(X)\right)$ and $C_{x}^{\Lambda}(Y)=C_{x}^{\Lambda}(X)$. Then we can replace $X$ by $Y$ and we are done by induction on the dimension.

Let $h: \overline{\mathscr{D}(X, x, \Lambda)} \rightarrow K$ be the deformation to $C_{x}^{\Lambda}(X)$. We assume $x=0$ in what follows, though we keep the notation $x$. Let $\mathscr{L}\left(C_{x}^{\Lambda}(X)\right)$ be

$$
C_{x}^{\Lambda}(X) \cap \bigcup_{i=0}^{e-1} S(0, i),
$$

where $e=\left[K^{\times}: \Lambda\right]$. We call $\mathscr{L}\left(C_{x}^{\Lambda}(X)\right)$ the $\Lambda$-link of $C_{x}^{\Lambda}(X)$. Note that the $\Lambda$-cone generated by $\mathscr{L}\left(C_{x}^{\Lambda}(X)\right)$ equals $C_{x}^{\Lambda}(X)$. Let $\tilde{\mathscr{L}}\left(C_{x}^{\Lambda}(X)\right)$ be $\mathscr{L}\left(C_{x}^{\Lambda}(X)\right) \times$ $(B(0, n) \cap \Lambda)$ for some ball $B(0, n)$ around 0 . Since there are definable choice functions, there is a map

$$
d: \tilde{\mathscr{L}}\left(C_{x}^{\Lambda}(X)\right) \rightarrow \overline{\mathscr{D}(X, x, \Lambda)}
$$


with $d(z, \lambda) \in h^{-1}(\lambda)$ for all $\lambda$ and $\lim _{\lambda \rightarrow 0} d(z, \lambda)=z$ for all $z$. Since we may and do suppose that $z \neq d(z, \lambda)$, the image of $d$ is of $\operatorname{dimension} \operatorname{dim}\left(\widetilde{\mathscr{L}}\left(C_{x}^{\Lambda}(X)\right)\right)=$ $\operatorname{dim}\left(\mathscr{L}\left(C_{x}^{\Lambda}(X)\right)\right)+1=\operatorname{dim}\left(C_{x}^{\Lambda}(X)\right)+1$. We send $d\left(\tilde{\mathscr{L}}\left(C_{x}^{\Lambda}(X)\right)\right)$ into $X$ by $r(z, \lambda)=\lambda \cdot z$ and we set $Y=r\left(d\left(\tilde{\mathscr{L}}\left(C_{x}^{\Lambda}(X)\right)\right)\right)$. Then $Y$ is a definable subset of $X$ of dimension $\operatorname{dim}\left(C_{x}^{\Lambda}(X)\right)$ and by construction $C_{x}^{\Lambda}(Y)=C_{x}^{\Lambda}(X)$.

\section{A local Crofton formula}

6.1. Local direct image. Let $p: X \rightarrow Y$ be a definable function between two definable sets of the same dimension $d$. If $\varphi$ is a function in $\mathscr{C}(X)$ and $y$ is in $Y$ we set $p_{!}(\varphi)(y)=\sum_{x \in p^{-1}(y)} \varphi(x)$ if $p^{-1}(y)$ is finite and $p_{!}(\varphi)(y)=0$ if it is infinite. The function $p_{!}(\varphi)$ lies in $\mathscr{C}(Y)$, since the cardinality of $p^{-1}(y)$ takes only finitely many values when $y$ runs over $Y$.

If $X$ is a definable subset of $K^{n}$ and $x$ is a point of $K^{n}$, we define the algebra $\mathscr{C}(X)_{x}$ of germs of constructible functions in $\mathscr{C}(X)$ at $x$ to be the quotient of $\mathscr{C}(X)$ by the equivalence relation $\varphi \sim \varphi^{\prime}$ if $\mathbf{1}_{B(x, n)} \varphi=\mathbf{1}_{B(x, n)} \varphi^{\prime}$ for $n$ large enough. That definition is only relevant when $x$ is in the closure of $X$. Also, if $\varphi$ is in $\mathscr{C}(X)_{x}$ is the germ of a locally bounded function $\psi, \Theta_{d}(\varphi):=\Theta_{d}(\psi)(x)$ does not depend on the representative $\psi$.

Let $p: K^{m} \rightarrow K^{d}$ be a linear projection and let $X$ and $Y$ be respectively definable subsets of $K^{m}$ and $K^{d}$ such that $p(X) \subset Y$. Fix $x$ in $K^{m}$. When the condition (*) is satisfied,

$$
\text { there exists } n \geq 0 \text { such that } p^{-1}(p(x)) \cap \overline{X \cap B(x, n)}=\{x\} \text {, }
$$

then, for every function $\varphi$ in $\mathscr{C}(X)$, the class of $p_{!}\left(\varphi \mathbf{1}_{B(x, n)}\right)$ in $\mathscr{C}(Y)_{p(x)}$ does not depend on $n$ for $n$ large enough. We denote it by $p_{!, x}(\varphi)$. We also denote by $p_{!, x}$ the corresponding morphism $\mathscr{C}(X)_{x} \rightarrow \mathscr{C}(Y)_{p(x)}$.

6.2. The local Crofton formula for the local density. For $x$ a point in $K^{n}$ we consider $K^{n}$ as a vector space with origin $x$ and for $0 \leq d \leq n$, we denote by $G(n, n-d)$ the corresponding Grassmannian of $(n-d)$-dimensional vector subspaces of $K^{n}$. It is a compact $K$-analytic variety, endowed with a unique measure $\mu_{n, d}$ invariant under $\mathrm{GL}_{n}(R)$ and such that $\mu_{n, d}(G(n, n-d))=1$.

For any $V$ in $G(n, n-d)$, we denote by $p_{V}: K^{n} \rightarrow K^{n} / V$, the canonical projection, where $K^{n} / V$ is identified with the $K$-vector space $K^{d}$. This identification enables the computation of the local density of germs in $K^{n} / V$.

Let $X$ be a definable subset of $K^{n}$ of dimension $d$ and let $x$ be a point of $K^{n}$. By general dimension theory for definable sets there exists a dense definable open subset $\Omega\left(=\Omega_{X}\right)$ of $G(n, n-d)$ such that for every $V$ in $\Omega$ the projection $p_{V}$ satisfies the condition $(*)$ with respect to $(X, x)$. 
The following statement is the $p$-adic analogue of the so-called local Crofton formula proved in [10] for real subanalytic sets and more generally in [11], again in the real subanalytic setting, in its multi-dimensional version.

6.2.1 Theorem. Let $X$ be a definable subset of $K^{n}$ of dimension $d$ and let $x$ be a point of $K^{n}$. Let $\varphi$ in $\mathscr{C}(X)_{x}$ be the germ of a locally bounded function. Then

$$
\Theta_{d}(\varphi)(x)=\int_{V \in \Omega \subset G(n, n-d)} \Theta_{d}\left(p_{V !, x}(\varphi)\right) d \mu_{n, d}(V) .
$$

We may assume that $X=\bar{X}$ by Proposition 2.4.1 and that $x=0$ and $0 \in X$, for if $0 \notin X, \Theta_{d}(X)(0)=\Theta_{d}\left(p_{V !, x}(\varphi)\right)=0$ (for generic $V$ ) and the statement of Theorem 6.2.1 is then true.

In order to emphasize the geometric-measure part of 6.2.1 we start with the following lemma, which is Theorem 6.2.1 for $X$ a definable $\Lambda$-cone of $K^{n}$ of dimension $d$ contained in some $d$-dimensional vector space of $K^{n}$ and $\varphi=\mathbf{1}_{X}$.

6.2.2 Lemma. Let $\Lambda \in \mathcal{D}, \Pi \in G(n, d)$ and $X$ be a definable $\Lambda$-cone contained in $\Pi$ and with origin 0 . Then

$$
\Theta_{d}(X)(0)=\int_{V \in \Omega \subset G(n, n-d)} \Theta_{d}\left(p_{V}(X)\right)\left(p_{V}(0)\right) d \mu_{n, d} .
$$

Proof. For every $V \in G(n, n-d)$, by linearity of $p_{V}, p_{V}(X)$ is a $\Lambda$-cone of $K^{n} / V$ with origin $p_{V}(0)$, and as $\operatorname{dim}(\Pi)=\operatorname{dim}\left(K^{n} / V\right), p_{V}(X)$ is isomorphic to $X$, for generic $V\left(V \in \Omega=\Omega_{\Pi}\right)$. In what follows we denote $p_{V}(0)$ by 0 . Take an integer $e>0$ such that $\pi_{K}^{e} \in \Lambda$, where we recall that $\pi_{K}$ is a uniformizer of $R$. The sets $X$ and $p_{V}(X)$ being $\Lambda$-cones, one has the following disjoint union relations

$$
\begin{gathered}
X=\coprod_{z \in \mathbb{Z}} \pi_{K}^{z e} \cdot\left(\coprod_{i=0}^{e-1} X \cap S(0, i)\right) \\
\text { and } p_{V}(X)=\coprod_{z \in \mathbb{Z}} \pi_{K}^{z e} \cdot\left(\coprod_{c=0}^{e-1}\left(p_{V}(X)\right) \cap S(0, c)\right)
\end{gathered}
$$

It follows by the definition of $\Theta_{d}$ that

$$
\Theta_{d}(X)(0)=\frac{\left(1-q^{-d}\right)^{-1}}{e} \sum_{i=0}^{e-1} q^{i d} \mu_{d}(X \cap S(0, i))
$$

and $\Theta_{d}\left(p_{V}(X)\right)(0)=\frac{\left(1-q^{-d}\right)^{-1}}{e} \sum_{c=0}^{e-1} q^{c d} \mu_{d}\left(p_{V}(X) \cap S(0, c)\right)$. 
For each $i=0, \ldots, e-1$, let $C_{i}$ be

$$
\coprod_{z \in \mathbb{Z}} \pi_{K}^{z e} \cdot p_{V}(X \cap S(0, i)) .
$$

One has $p_{V}(X)=\coprod_{i} C_{i}$ by the linearity of $p_{V}$, and, the $C_{i}$ are definable since $X$ is a $\Lambda$-cone. Define the disjoint definable sets $A_{c}^{i}$, for $i$ and $c$ going from 0 to $e-1$, by

$$
A_{c}^{i}=C_{i} \cap S(0, c)
$$

Clearly

$$
\coprod_{i=0}^{e-1} A_{c}^{i}=p_{V}(X) \cap S(0, c) .
$$

Moreover, the sets $\pi_{K}^{i-c} \cdot A_{c}^{i}$ are disjoint by linearity of $p_{V}$ and by bijectivity of $p_{V}$ on $\Pi$. By the fact that

$$
q^{c d} \mu_{d}\left(A_{c}^{i}\right)=q^{i d} \mu_{d}\left(\pi_{K}^{i-c} \cdot A_{c}^{i}\right)
$$

we obtain

$$
\begin{aligned}
\sum_{c=0}^{e-1} q^{c d} \mu_{d}\left(p_{V}(X) \cap S(0, c)\right) & =\sum_{c=0}^{e-1} q^{c d} \sum_{i=0}^{e-1} \mu_{d}\left(A_{c}^{i}\right) \\
& =\sum_{i=0}^{e-1} q^{i d} \mu_{d}\left(\coprod_{c=0}^{e-1} \pi_{K}^{i-c} \cdot A_{c}^{i}\right) \\
& =\sum_{i=0}^{e-1} q^{i d} \mu_{d}\left(B_{V}^{i}\right),
\end{aligned}
$$

where $B_{V}^{i}:=\coprod_{c=0}^{e-1} \pi_{K}^{i-c} \cdot A_{c}^{i}$. Let us now consider $\Phi_{V}: \Pi \backslash\{0\} \rightarrow\left(K^{n} / V\right) \backslash\{0\}$, defined by

$$
\Phi_{V}(x)=\pi_{K}^{\operatorname{ord}(x)-\operatorname{ord}\left(p_{V}(x)\right)} \cdot p_{V}(x) .
$$

This map is bijective from $X \cap S(0, i)$ to $B_{V}^{i}$, since $p_{V}$ is bijective from $X$ to $p_{V}(X)$. By change of variables one obtains

$$
\mu_{d}\left(B_{V}^{i}\right)=\int_{X \cap S(0, i)}\left|\operatorname{Jac}\left(\Phi_{V}\right)\right| d \mu_{d}
$$

Furthermore, by Fubini,

$$
\int_{V \in \Omega} \mu_{d}\left(B_{V}^{i}\right) d \mu_{n, d}=\int_{x \in X \cap S(0, i)} \int_{V \in \Omega}\left|\operatorname{Jac}\left(\Phi_{V}\right)(x)\right| d \mu_{n, d}(V) d \mu_{d}(x) .
$$


Note that, for $x \in S(0, i)$, the quantity $\kappa_{i}=\int_{V \in \Omega}\left|\operatorname{Jac}\left(\Phi_{V}\right)(x)\right| d \mu_{n, d}(V)$ does not depend on $x$. Indeed, $\mathrm{GL}_{n}(R)$ acts transitively on $S(0, i), \mu_{n, d}$ is invariant under this action and if $g \in \mathrm{GL}_{n}(R)$ and $x^{\prime}=g \cdot x$ for $x, x^{\prime} \in S(0, i)$, then $\operatorname{Jac}\left(\Phi_{V}\right)\left(x^{\prime}\right)=\operatorname{Jac}\left(\Phi_{g-1 \cdot V}\right)(x)$. Moreover, by linearity of $p_{V}$, one has that $\kappa_{i}=\kappa$ is independent of $i$. It follows from (6.2.2), (6.2.3) and (6.2.4) that

$$
\begin{gathered}
\int_{V \in \Omega} \Theta_{d}\left(p_{V}(X)\right)(0) d \mu_{n, d}(V)=\frac{\left(1-q^{-d}\right)^{-1}}{e} \sum_{i=0}^{e-1} q^{i d} \int_{x \in X \cap S(0, i)} \kappa d \mu_{d}(x) . \\
=\kappa \cdot \frac{\left(1-q^{-d}\right)^{-1}}{e} \sum_{i=0}^{e-1} q^{i d} \mu_{d}(X \cap S(0, i)) .
\end{gathered}
$$

Finally, by (6.2.1), we obtain

$$
\int_{V \in \Omega} \Theta_{d}\left(p_{V}(X)\right)(0) d \mu_{n, d}(V)=\kappa \cdot \Theta_{d}(X)(0) .
$$

One gets $\kappa=1$ by taking $X=\Pi$ in the latter formula.

Lemma 6.2.2 may be viewed as the tangential formulation of the local Crofton formula for general definable $\Lambda$-cone sets and its proof captures its geometric measure content. Note that its proof still works assuming that $X$ is a definable $\Lambda$-cone of dimension $d$ in $K^{n}$, instead of a definable $\Lambda$-cone of dimension $d$ contained in some $d$-dimensional vector space $\Pi$. Indeed, it is essentially enough to replace, in the proof of Lemma 6.2.2, $\Phi_{V}: \Pi \backslash\{0\} \rightarrow\left(K^{n} / V\right) \backslash\{0\}$ by the restriction of the mapping $w \mapsto \Psi_{V}(x)=\pi_{K}^{\operatorname{ord}(x)-\operatorname{ord}\left(p_{V}(x)\right)} p_{V}(x)$ on the smooth part of $X$ (the fibers of $\Psi_{V \mid X}$ being counted with multiplicity in the area formula). Hence we get the following extension of Lemma 6.2.2:

6.2.3 Lemma. Let $\Lambda \in \mathscr{D}$ and $X$ be a definable $\Lambda$-cone of $K^{n}$ with origin 0 . Then

$$
\Theta_{d}(X)(0)=\int_{\Omega \subset G(n, n-d)} \Theta_{d}\left(p_{V !, 0}\left(\mathbf{1}_{X}\right)\right) d \mu_{n, d}(V) .
$$

6.2.4 Remark. For $V \in G(n, n-d)$ and $y \in\left(K^{n} / V\right) \backslash\{0\}$, let us denote by $\tilde{V}_{y}$ the fiber $\Psi_{V}^{-1}(\{y\})$ of $\Psi_{V}: K^{n} \backslash V \rightarrow\left(K^{n} / V\right) \backslash\{0\}$, where $\Psi_{V}(x)=$ $\pi_{K}^{\operatorname{ord}(x)-\operatorname{ord}\left(p_{V}(x)\right)} p_{V}(x)$. Note that $\tilde{V}_{y} \subset S(0$, ord $y) \backslash V$ and $\operatorname{GL}_{n}(R)$ acts on $\widetilde{V}=\left\{\tilde{V}_{y} \mid V \in G(n, n-d), y \in\left(K^{n} / V\right) \backslash\{0\}\right\}$. For $X$ a definable set of dimension $d$ in $S(0, c)$, where $c \in \mathbb{Z}$, the statement of Lemma 6.2 .3 may be reformulated as

$$
\mu_{d}(X)=\int_{V \in \Omega} \int_{y \in S(0, c) \subset K^{n} / V} \#\left(X \cap \tilde{V}_{y}\right) d \mu_{d}(y) d \mu_{n, d}(V) .
$$


Now note that the mapping $(V, y) \mapsto \widetilde{V}_{y}$ defined from $\{(V, y) \mid V \in G(n, n-d), y \in$ $\left.K^{n} / V\right\}$ to $\tilde{V}$ is one-to-one and that the image of the Haar measure of $\mathrm{GL}_{n}(R)$ under $g \mapsto g \cdot \widetilde{V}_{0}$ (for $\widetilde{V}_{0}$ fixed in $\widetilde{V}$ ) gives a $\operatorname{GL}_{n}(R)$-invariant measure $v$ on $\widetilde{V}$ such that for $E \subset \tilde{V}, E$ subanalytic say, we have

$$
v(E)=\int_{V \in \Omega} \int_{y \in S(0, c) \subset K^{n} / V} \mathbf{1}_{E}\left(\tilde{V}_{y}\right) d \mu_{d}(y) d \mu_{n, d}(V) .
$$

To obtain the above equality, it is enough to remark that the right hand side gives a function on subsets of $\widetilde{V}$ which pulls back on $\mathrm{GL}_{n}(R)$ as a Haar measure. With these notations we see that Lemma 6.2.3 is nothing else than the classical spherical Crofton formula for $X \cap S(0, c)$ (for a standard reference see [24], Theorem 3.2.48 and note that the proof may be applied in our setting):

6.2.5 Theorem. Let $X$ be a definable set of $S(0,0) \subset K^{n}$ of dimension $d$, then

$$
\begin{gathered}
\mu_{d}(X)=\int_{V \in \Omega} \int_{y \in S(0,0) \subset K^{n} / V} \#\left(X \cap \tilde{V}_{y}\right) d \mu_{d}(y) d \mu_{n, d}(V) \\
=\int_{\tilde{v} \in \tilde{V}} \#(X \cap \tilde{v}) d v(\tilde{v}) .
\end{gathered}
$$

For the general setting we will use the following auxiliary lemma.

6.2.6 Lemma. Let $\Lambda$ be in $\mathcal{D}$ and let $X \subset K^{n}$ be a definable set of dimension $d$. Suppose that $p: K^{n} \rightarrow K^{d}$ is a coordinate projection which is injective on $X$. Then there exist definable sets $C_{j}$ of dimension $<d$ and a finite partition of $X$ into definable parts $X_{j}$ such that $p$ is injective on $C_{0}^{\Lambda}\left(X_{j}\right) \backslash C_{j}$ for each $j$.

Proof. Since $C_{0}^{\Lambda}(X) \subset C_{0}^{K^{\times}}(X)$ for any $\Lambda$ in $\mathscr{D}$, we may suppose that $\Lambda=K^{\times}$. We may also suppose that $0 \in \bar{X} \backslash X$. Partition $C_{0}^{K^{\times}}(X)$ into finitely many definable parts $B_{j}$ such that $p$ is injective on each set $B_{j}$. By linearity of $p$ we may suppose that each $B_{j}$ is a $K^{\times}$-cone. For each $j$ let $B_{j}^{\prime}$ be the definable subset of $K^{n}$ consisting of the union of all lines $\ell \in K^{n}$ through 0 such that the distance between $\ell \cap S(0,0)$ and $B_{j} \cap S(0,0)$ is strictly smaller than the distance between $\ell \cap S(0,0)$ and $B_{i} \cap S(0,0)$ for all $i \neq j$. Put $X_{j}^{0}:=X \cap B_{j}^{\prime}$ for each $j$, and take a finite definable partition of $X$ into parts $X_{j}$ satisfying $X_{j}^{0} \subset X_{j}$ for each $j$. By construction $C_{0}^{K^{\times}}\left(X_{j}\right)=$ $C_{0}^{K^{\times}}\left(X_{j}^{0}\right) \subset \overline{B_{j}}$. Let $C_{j}$ be $\overline{B_{j}} \backslash B_{j}$. Then the $X_{j}$ and $C_{j}$ are as desired.

We now prove Theorem 6.2.1 in its general setting, that is to say, for $X$ a given definable subset of $K^{n}$ of dimension $d$ instead of some definable $\Lambda$-cone of $K^{n}$ as in Lemma 6.2.3. 
Proof of Theorem 6.2.1. As in the proof of Theorem 3.6.2 we may assume that $\varphi=\mathbf{1}_{X}$. Up to a finite partition of $X$ into definable parts we may suppose that $X$ is the graph of an $\varepsilon$-analytic map $U \subset K^{d} \rightarrow K^{n-d}$ as in Corollary 5.3.8 and then it follows by this corollary that

$$
\Theta_{d}(X)(0)=\Theta_{d}\left(S C_{0}^{\Lambda}(X)\right)(0)=\Theta_{d}\left(C_{0}^{\Lambda}(X)\right)(0) .
$$

For $C_{0}^{\Lambda}(X)$ we know that Theorem 6.2.1 holds by Lemma 6.2.3, that is

$$
\Theta_{d}\left(C_{0}^{\Lambda}(X)\right)(0)=\int_{V \in \Omega \subset G(n, n-d)} \Theta_{d}\left(p_{V !, 0}\left(\mathbf{1}_{C_{0}^{\Lambda}(X)}\right) d \mu_{n, d}(V) .\right.
$$

We claim that, for generic $V$,

$$
\Theta_{d}\left(p_{V !, 0}\left(\mathbf{1}_{C_{0}^{\Lambda}(X)}\right)\right)=\Theta_{d}\left(p_{V !, 0}\left(\mathbf{1}_{X}\right)\right)
$$

which finishes the proof. We prove the claim as follows. Fix $V$. By Lemma 6.2.6 we can partition $X$ into finitely many definable parts $X_{j}$ (depending on $V$ ) such that $p_{V}$ is injective on $X_{j}$ and, up to a definable set of dimension $<d$, also on $C_{0}^{\Lambda}\left(X_{j}\right)$. By additivity it is now enough to prove that

$$
\Theta_{d}\left(p_{V}\left(C_{0}^{\Lambda}\left(X_{j}\right)\right)\right)(0)=\Theta_{d}\left(p_{V}\left(X_{j}\right)\right)(0),
$$

which follows from Theorem 3.6.2 for open sets since $p_{V}\left(C_{0}^{\Lambda}\left(X_{j}\right)\right)=C_{0}^{\Lambda}\left(p_{V}\left(X_{j}\right)\right)$.

\section{References}

[1] K. Bekka, Regular stratification of subanalytic sets. Bull. London Math. Soc. 25 (1993), 7-16. Zbl 0826.32004 MR 1190357

[2] R. Cluckers, Presburger sets and p-minimal fields. J. Symbolic Logic 68 (2003), 153-162. Zbl 1046.03019 MR 1959315

[3] R. Cluckers, Analytic p-adic cell decomposition and integrals. Trans. Amer. Math. Soc. 356 (2004), 1489-1499. Zbl 1048.11094 MR 2034315

[4] R. Cluckers, Multi-variate Igusa theory: Decay rates of $p$-adic exponential sums. Internat. Math. Res. Notices 76 (2004), 4093-4108. Zbl 1063.11047 MR 2109987

[5] R. Cluckers, G. Comte and F. Loeser, Lipschitz continuity properties for $p$-adic semialgebraic and subanalytic functions. Geom. Funct. Anal. 20 (2010), 68-87.Zbl 1220.12003 MR 2647135

[6] R. Cluckers, L. Lipshitz and Z. Robinson, Analytic cell decomposition and analytic motivic integration. Ann. Sci. École Norm. Sup. 39 (2006), 535-568. Zbl 1168.12006 MR 2290137

[7] R. Cluckers and L. Lipshitz, Fields with analytic structure. J. Eur. Math. Soc. (JEMS) 4 (2011), 1147-1223. Zbl 05919474 MR 2800487 
[8] R. Cluckers and F. Loeser, Constructible motivic functions and motivic integration. Invent. Math. 173 (2008), 23-121. Zbl 1179.14011 MR 2403394

[9] P. J. Cohen, Decision procedures for real and p-adic fields. Comm. Pure Appl. Math. 22 (1969), 131-151. Zbl 0167.01502 MR 0244025

[10] G. Comte, Équisingularité réelle : nombres de Lelong et images polaires. Ann. Sci. École Norm. Sup. 33 (2000), 757-788. Zbl 0981.32018 MR 1832990

[11] G. Comte and M. Merle, Équisingularité réelle II : invariants locaux et conditions de régularité. Ann. Sci. École Norm. Sup. 41 (2008), 221-269. Zbl 1163.32012 MR 2468482

[12] G. Comte, J.-M. Lion and J.-P. Rolin, Nature log-analytique du volume des sousanalytiques. Illinois J. Math. 44 (2000), 884-888. Zbl 0982.32009 MR 1804313

[13] J. Denef, The rationality of the Poincaré series associated to the $p$-adic points on a variety. Invent. Math. 77 (1984), 1-23. Zbl 0537.12011 MR 0751129

[14] J. Denef, On the evaluation of certain $p$-adic integrals. In Séminaire de théorie des nombres, Paris 1983-84, Progr. Math. 59, Birkhäuser, Boston, Mass., 1985, 25-47. Zbl 0597.12021 MR 0902824

[15] J. Denef, p-adic semi-algebraic sets and cell decomposition. J. Reine Angew. Math. 369 (1986), 154-166. Zbl 0584.12015 MR 0850632

[16] J. Denef, Degree of local zeta functions and monodromy. Compositio Math. 89 (1993), 207-216. Zbl 0932.11073 MR 1255694

[17] J. Denef and L. van den Dries, p-adic and real subanalytic sets. Ann. of Math. 128 (1988), 79-138. Zbl 0693.14012 MR 0951508

[18] J. Denef and F. Loeser, Motivic Igusa zeta functions. J. Algebraic Geom. 7 (1998), 505-537. Zbl 0943.14010 MR 1618144

[19] J. Denef and F. Loeser, Germs of arcs on singular algebraic varieties and motivic integration. Invent. Math. 135 (1999), 201-232. Zbl 0928.14004 MR 1664700

[20] R. Draper, Intersection theory in analytic geometry. Math. Ann. 180 1969, 175-204. Zbl 0157.40502 MR 0247134

[21] L. van den Dries, D. Haskell and D. Macpherson, One-dimensional $p$-adic subanalytic sets. J. London Math. Soc. 59 (1999), 1-20. Zbl 0932.03038 MR 1688485

[22] L. van den Dries and P. Scowcroft, On the structure of semi-algebraic sets over $p$-adic fields. J. Symbolic Logic 53 (1988), 1138-1164. Zbl 0692.14014 MR 0973105

[23] L. van den Dries, Dimension of definable sets, algebraic boundedness and Henselian fields. Ann. Pure Appl. Logic 45 (1989), 189-209. Zbl 0704.03017 MR 1044124

[24] H. Federer, Geometric measure theory. Grundlehren Math. Wiss. 153, Springer-Verlag, Berlin 1969. Zbl 0176.00801 MR 0257325

[25] D. Heifetz, p-adic oscillatory integrals and wave front sets. Pacific J. Math. 116 (1985), 285-305. Zbl 0528.22008 MR 0771637

[26] J. P. Henry, M. Merle and C. Sabbah, Sur la condition de Thom stricte pour un morphisme analytique complexe. Ann. Sci. École Norm. Sup. 17 (1984), no. 2, 227-268. Zbl 0551.32012 MR 0760677

[27] H. Hironaka, Stratification and flatness. In Real and complex singularities (Proc. Ninth Nordic Summer School/NAVF Sympos. Math., Oslo, 1976), Sijthoff and Noordhoff, Alphen aan den Rijn 1977, 199-265. Zbl 0424.32004 MR 0499286 
[28] T. C. Kuo, The ratio test for analytic Whitney stratifications. In Proceedings of Liverpool Singularities Symposium I, Lecture Notes in Math. 192, Springer-Verlag, Berlin 1971, 141-149. Zbl 0246.32006 MR 0279333

[29] K. Kurdyka, On a subanalytic stratification satisfying a Whitney property with exponent 1. In Real algebraic geometry (Rennes, 1991), Lecture Notes in Math. 1524, Springer-Verlag, Berlin 1992, 316-322. Zbl 0779.32006 MR 1226263

[30] K. Kurdyka and A. Parusiński, $w_{f}$-stratification of subanalytic functions and the Lojasiewicz inequality. C. R. Acad. Sci. Paris Sér. I Math. 318, no. 2, (1994), 129-133. Zbl 0799.32007 MR 1260324

[31] K. Kurdyka, J.-B. Poly and G. Raby, Moyennes des fonctions sous-analytiques, densité, cône tangent et tranches. In Real analytic and algebraic geometry (Trento, 1988), Lecture Notes in Math. 1420, Springer-Verlag, Berlin 1990, 170-177. Zbl 0694.32001 MR 1051211

[32] K. Kurdyka and G. Raby, Densité des ensembles sous-analytiques. Ann. Inst. Fourier 39 (1989), 753-771. Zbl 0673.32015 MR 1030848

[33] P. Lelong, Intégration sur un ensemble analytique complexe. Bull. Soc. Math. France 85 (1957), 239-262. Zbl 0079.30901 MR 0095967

[34] J.-M. Lion, Densité des ensembles semi-pfaffiens. Ann. Fac. Sci. Toulouse Math. 7 (1998), 87-92. Zbl 0917.32003 MR 1658448

[35] T. L. Loi, Thom stratifications for functions definable in o-minimal structures on $(\mathbb{R},+, \cdot)$. C. R. Acad. Sci. Paris Sér. I Math. 324 (1997), no. 12, 1391-1394. Zbl 0883.32006 MR 1457093

[36] T. L. Loi, Verdier and strict Thom stratifications in o-minimal structures. Illinois J. Math. 42 (1998), no. 2, 347-356. Zbl 0909.32008 MR

[37] J. Oesterlé, Réduction modulo $p^{n}$ des sous-ensembles analytiques fermés de $\mathbb{Z}_{p}^{N}$. Invent. Math. 66 (1982), 325-341. Zbl 0473.12015 MR 0656627

[38] W. Pawłucki, Le théorème de Puiseux pour une application sous-analytique. Bull. Polish Acad. Sci. Math. 32 (1984), no. 9-10, 555-560. Zbl 0574.32010 MR 0782936

[39] J.-P. Serre, Quelques applications du théorème de densité de Chebotarev. Inst. Hautes Études Sci. Publ. Math. 54 (1981), 323-401. Zbl 0496.12011 MR 0644559

[40] Y. T. Siu, Analyticity of sets associated to Lelong numbers and the extension of closed positive currents. Invent. Math. 27 (1974), 53-156. Zbl 0289.32003 MR 0352516

[41] P. Thie, The Lelong number of a point of a complex analytic set. Math. Ann. 172 (1967), 269-312. Zbl 0158.32804 MR 0214812

[42] R. Thom, Ensembles et morphismes stratifiés. Bull. Amer. Math. Soc. 75 (1969), 240-284 Zbl 0197.20502 MR 0239613

[43] J. L. Verdier, Stratifications de Whitney et théorème de Bertini-Sard. Invent. Math. 36 (1976), 295-312. Zbl 0333.32010 MR 0481096

[44] H. Whitney, Tangents to an analytic variety. Ann. of Math. 81 (1965), 496-549. Zbl 0152.27701 MR 0192520

Received November 11, 2009 
Raf Cluckers, Université Lille 1, Laboratoire Painlevé, CNRS - UMR 8524, Cité Scientifique, 59655 Villeneuve d'Ascq Cedex, France, and Katholieke Universiteit Leuven,

Department of Mathematics, Celestijnenlaan 200B, 3001 Leuven, Belgium

E-mail: raf.cluckers@ @is.kuleuven.be

http://www.wis.kuleuven.be/algebra/Raf/

Georges Comte, Laboratoire J.-A. Dieudonné, Université de Nice - Sophia Antipolis, Parc Valrose, 06108 Nice Cedex 02, France (UMR 6621 du CNRS)

E-mail: georges.comte@univ-savoie.fr

http://www-math.unice.fr/membres/comte.html

François Loeser, Institut de Mathématiques de Jussieu, UMR 7586 du CNRS, Université Pierre et Marie Curie, 75005 Paris, France

E-mail: loeser@math.jussieu.fr

http://www.math.jussieu.fr/ loeser/ 